This report was prepared as an account of work sponsored by an agency of the United States Government. Neither the United States Government nor any agency thereol, nor any of their ermployees, makes any wilranty, express or implied, or assumes any legal liability or responsibility for the accuracy, completeness, or usefulness of any infortnation, apparatus, product, or process disclosed, or represents that its use would not infringe privalely owned rights. Reference herein to any specific commercial product, process, of service hy trade name, trademark, manufacturer, or otherwise does not necessarily constitute or imply its endorsement. recommendation, or favoring by the United States Government or iny agency thereof. The views and opinions of authors expressed herein do not necessarily state or reflect those of the United Siates Government or any agency thereor.

\title{
Improved rigorous upper bounds for transport due to passive advection described by simple models of bounded systems \\ PPPL-- ; $; 42$
}

\author{
Chang-Bae Kim² and John A. Krommes ${ }^{2}$
}

DE88 015821

\begin{abstract}
The work of hrommes and Smith on rigorous upper bounds for the turbulent transport of a passively advected scalar 'Ann. Phys. 177:2 i (1987)! is extended in two directions: (1) For their "reference model," int. sroved upper bounds are obtained by utilizing more sophisticated two-time onstraints which include the effects of cross-correlations up to fourth order. umerical solutions of the model stochastic differential equation are also obtanned; they show that the new bounds compare quite favorably with the exact results, even at large Reynolds and Kubo numbers. (2) The theory is extended to take account of a finite spatial autocorrelation length $L_{c}$. As a reasonably generic example, the problem of particle transport due to statistically specified stochastic magnetic fields in a collisionless turbulent plasma is revisited. $A$ bound is obtained which reduces for small $L_{c}$ to the quasilinear limit and for large $L_{c}$ to the strong turbulence limit, and which provides a reasonable and rigorous interpolation for intermediate values of $L_{c}$.
\end{abstract}

KEY WORDS: Passive advection, bounds, optimum theory, stochastic magnetic fields, transport, variational principles.

1 Work supported by U.S. D.o.E. Contract No. DE-AC02-76-CHO-3073.

2 Princeton University, Plasma Physics Laboratory, P.O. Box 451, Princeton, NJ 08543. 


\section{INTRODUCTION}

In this paper we explore the derivation of rigorous yet useful upper bounds for steadystate transport due to random passive advection.

It has long been recognized that the problem of obtaining an analytic theory of turbulence in fluids, plasmas, or similar nonlinear systems is extremely difficult. Csually, a statistical description is sought; in particular, one is often interested in the turbulent transport of various quantities such as particles or heat, which are described by certain twopoint cross-correlation functions. However, straightforward averaging procedures applied directly to the nonlinear equations of motion encounter immediat ely the closure problemnamely, lower-order moments are driven by higher-order ones, and a useful expression for the higher-order moments in terms of lower-order ones is not readily apparent. Sophisticated closures such as the direct-interaction approximation ${ }^{(1.2)}$ provide such expressions, but the resulting equations are extremely complicated and are very difficult to solve either analytically or numerically. Furtherrnore, such closures misrepresent certain aspects of the physics; for example, they are largely ignorant of mechanisms of instability and coherent structures, if they exist.

An alternative approach to the problem of turbulent transport is the so-called "optimum theory." (3,4) Although this method also uses statistical techniques, it is an approach quite different from the conventional closure-theoretical one. Instead of approximating the higher-order moments in terms of lower-order moments in order to close the moment hierarchy, the optimum theory employs a rigorous variational principle to address the question: What is the maximum attainable flux of some appropriate quantity, with given parameters and boundary conditions, subject tn various constraints derived from the equations of motion. Although this question differs from the one usually addressed by conventional statistical closures, which try to predict the flux as accurately as possible, it is perfectly legitimate, since physically the knowledge of an upper bound certainly helps one to estimate the true answer, even if crudely. Furthermore, if one can exhibit one combination of constraints which yields a finite bound, he is guararteed that adding an additional constraint will lead to a new bound which will be no worse than the old one. Thus one can in principle envision a sequence of bounds, obtained by adding more and more constraints, which converges to the true answer. In practice, of course, only a few constraints will be tractable. The principal goal of the present work, as was true also of Ref. 4, is to obtain experience with various constraints applied to simple yet nontrivial situations in order to better understand the utility of the method.

The original motivation for the optimum theory lay in Malkus' speculation ${ }^{(5)}$ that the physical state achieved in experiments of steady-state thermal convection corresponds to the mathematical solution which maximizes the heat flux. Although this turned out to be true only in certain limiting cases, it motivated Howard ${ }^{(0)}$ to ask a different, a]though mathematically rigorous, question: Instead of attempting to characterize or ascertain the true (physical) flux, he asked what the maximum possible flux would be, compatible with 
one or more coisstraints derived rigorously from the equations of motion. He showed how to formulate this question as a rigorous variational principle. Busse called the procedure the "optimum theory" and reviewed ${ }^{(3)}$ developments and applications of the method through 1978. Smith introduced the optimum theory to the plasma physics community with his discussion $^{\bar{T}}$ of transpon due to collisional drift waves. All those calculations were concerned with self-consistent problems. where all dependent variables are related to each uther.

Krommes and Smith pointed out ${ }^{(4)}$ that problems of passive advection, where the advecting velocity. field is statjstically specified instead of self-consistently determined, are also of interest. Tot only do passive problems arise in various physical applications. an equally important motivation for studfing them is that their reduced mathematical complexity enables one to exhibit and understand certain general characteristics of the optimum theory much more simply. Krommes and Smith considered in detail a pedagogical "reference model" for which an exact solution is avalable. They also discussed general features of the generic optimum problem of passive advection under the so-called "basic" constraint. However, they noted that passive problems involve one new qualitative feature-namely, a new dimensionless variable. the Kubo number, ${ }^{(\mathbf{8}, 2)}$ must be introduced in order to describe the statistical nature of the temporal and spatial variation of the specified advecting field. Krommes and Smith emphasized that the effects of finite autocorrelation time and length described by the Kubo number were not captured by the basic constraint which had been used exclusively in the self-consistent problems, and they showed how to incorporate these effects into the formalism.

Although the detailed mathematical features of their work are described in Sec. 2, it is useful to give a few introductory remarks here. They considered the situation in which a scalar field $T$ is passively advected by a random velocity field $u$, dependent only on time. in the presence of dissipation and in a bounded region. Thus the model equation is of diffusion type with an advection term which is stochastically, not dynamically, nonlines 1 ) The (time-independent) boundary conditions for the scalar field were taken to be statistically sharp: $T=1$ at $x=0$ and $T=0$ at $x=1$. Physically, this mak's the walls infinite sources or sinks, which is sometimes appropriate in physical applications. The turbulence was assumed to be statistically stationary in time. They then proceeded as follows: First, they obtained the "basic" constraint by constructing a quadratic functional of $\delta T(x, t)$ from the global balance of scalar variance ("energy") $\left\langle\delta T^{2}(x, t)\right) / 2$. This is a standard way of finding an "action" or Lagrangian. Then, they pointed out that this constraint does not involve the Kubo number since it is constructed from moments at one point in space and time, so correlation lengths or times (two-point quantities) cannot enter. Hence the basic bound, although rigorous, behaves as though the Kubo number were infinite. In order to incorporate the effects of finite correlations, they const ructed a two-time constraint by multiplying the equation for the fuctuations $\delta T(x, t)$ by $\delta u\left(t^{\prime}\right)$ at a different time, averaging over the ensemble, and integrating over $x$. They showed that the solution of the resulting Euler-Lagrange equation not only reduces as expected to the result of the basic bound in the limit $K \rightarrow \infty$ of strong turbulence, it also reproduces correctly the exact, quasilinear, 
result as $K-0$. In fact. for the reference model the new bound scales correctly in all regimes of Reynolds number and kubo number.

For the reference model. the basic bound (or the two-time bound for $h=x$ ) is no more than about $25 \%$ larger than the 1 rue answer in the worst case of infinite Rernolds number (very strong turbulence). and can be obtained quite simply. The direct-interaction approximation predicts a closer result ${ }^{(4)}$ (lower than the exact answer) but requires substantial computational labor to obtain. Thus an advantage of the optimum theory over conventional closures can be relatively simple algetra or numerical computations. The advantage is not so clear when two-time constraints are considered. although the rigor of the optimum theory is very appealing.

Of course, there are an infinite number of two-point constraints. Although the constraint of Krommes and Smith may well be the simplest, it is certainly not unique. One of our purposes in this paper is to explore the possibility of improving the upper bounds by adding more constraints. This can be done by including the effects of triplet andior higher-order cross-correlations in the formulation. Indeed, we find that the upper bounds can be improved, although only after lengthy algebra. The must successful constraint we have found involves fourth-order correlations.

Although Krommes and Smith were able to obtain simply the exact result for the flux predicted by the reference model in the limit $K \rightarrow \infty$ (because that limit involves no time dependence), they did not at tempt to solve the model for finite $K$. In the present work we accomplish this solution by statistically averaging an ensemble of numerical solutions of the stochastic differential equation. This allows us to demonstrate that the improved bounds compare quite favorably with the true solution.

One flaw of the present calculations based on the reference model is that since there the advecting velocity field depends only on time-i.e., the correlation length is infinitevarious analytic shortcuts can be taken and one worries that the manipulations may not generalize readily to the physically interesting case of finite correlation lengths. Thus in the second part of this paper we generalize the work of Krommes and Smith by studying a system where the effects of the finite correlation lengths are important. In particular, we revisit the problem of particle transport in a turbulent plasma due to stochastic magnetic fields. This problem has been treated extensively in the literature (see, for example, Refs. 9,10 , and 11 ), since field line stochasticity has been advanced ${ }^{(9)}$ as a likely mechanism to partly explain the anomalous transport of electrons and heat in a turbulent, magnetically confined fusion plasma. Most of the works so far can be classified as closure-theoretical approximations to the passive situation, ${ }^{(11)}$ although recently there have been several attempts to study the self-consistent problem. ${ }^{(12-14)}$ Krommes and Smith did consider this problem as an application of the optimum theory; however, they only considered the basic constraint, which behaves as though the correlation time and lengths are infinite. We shall show that it is, in fact, possible to generalize their calculation to include both finite correlation time and, in particular, finite correlation lengths. 
The rest of this paper is organized as follows. In Sec. 2. We show how wo improve the two-time result of krommes and Smith for the reference model by taking into account hiather-rorder coriss-correlations. Twu ways of improvement are pursued. First. while hromunes and Smith annihilate a triplet cross-correlation by integrating the two-time constraint wer $x$. we simply keep that term by not integrating. which leads us to introduce a spaceat well as cime-dependent Lasrange multiplier. The resulting bound is found tu be inprowed =lightl: for finite $K u b u$ number, but not improved in the limiting cases of ejther ecr. " in inite kubo number. The reason is that in the case of vanishing kubo number buth two-time bounds are exact (quasilinear). while in the case of infinite hubo number bun in tur-time constraints reduce to the basic, one-time constraint and are thus redundant. Second. We show how to improve the bounds in the cases of infinite as well as finite Kubo number by adding to the constraints of hrommes and Smith another two-time constraint cunstructed from a fourth-order cross-correlation. The application to the stochastic field problem is described in Sec. 3. We discuss our results in Sec. 4. In Appendix A we describe ar numerical solutions of the generalized reference model. In Appendix B we give a brief discussion of functional derivatives on bounded spatial domains. Finally, in Appendix $\mathrm{C}$ w'e give the detailed derivation of an optimum equation for (collisionless) transport due to stuchastic magnetic fields.

\section{IMPROVED UPPER BOUNDS FOR THE REFERENCE MODEL OF KROMMES AND SMITH}

We begin this section by defining the problem which Krommes and Smith called the reference model In this pedagogical problem, a scalar field $T$ is passively advected by a centered. time-stationary. Gaussian velocity field $u(t)$ whose statistics are specified explicitly in a one-dimensional, bounded region, in the presence of (classical) dissipation. The imposed boundary conditions are statistically sharp: $T=1$ at $x=0$ and $T=0$ at $x=1$; these are sometimes called "rigid" because the fluctuation $\delta T$ vanishes at the boundaries. This model serves as a tractable prototype for a wide variety of advection probiems. The purpose of studying the very simple reference model is that in certain limits an exacr solution is available ${ }^{(4)}$ to which the results of the variational calculation can be compared: also, while in more complicated, physical situations the Euler-Lagrange equation must be solved numerically, for the reference model much of the algebra can be done analytically:

For purposes of clarity and comparison. we shall follow closely the notations of Krommes and Smith. Then the evolution equation of $T$ is

$$
\frac{\partial T}{\partial t}-u(t) \frac{\partial T}{\partial x}-R^{-1} \frac{\partial^{2} T}{\partial x^{2}}=0,
$$

where $R$ is the Reynolds number and all the variables have been made dimensionless: $u$ is normalized to $\bar{u} \doteq\left\langle u^{2}\right\rangle^{1 / 2}, x$ is normalized to the width $L$ of the region and thus is defined on the interval $[0,1]$, and the time $t$ is nomalized to the eddy turnover time $\tau_{L} \doteq L / \bar{u}$. 
As Krommes and Smith discussed. there are three fundamental time scales: in addition to the eddy tumover time $\tau_{L} !=1$ in dimensionless units), there is the hydrodynamic time $r_{r}=R$ and the autocorrelation time $\tau_{a r}=K$. While the first two time scales are obtained by balancing the first term of Eq. (1) with the second and third terms. respectively: the autocorrelation time enters eza the statistics of $u$. By means of dimensional analysis and random walk arguments, Krommes and Smith obtained several possible scalings for the dimensionless flux $\gamma$. namely classical $\left(\neg \mathrm{cl} \sim R^{-1}\right)$. quasilinear $\left(\sim_{q} \sim K\right)$. hydrodynamic $\left(-i_{1}-R\right)$. and strong turbulence $\left(\gamma_{\mathrm{s}}-1\right)$.

In the limit $K=\infty$. time dependence can be ignored in Eq. (1) and one ubtains a second-order ordinary differential equation which can be solved analyuically ${ }^{(t)}$ for each realization of the Gaussian random number $u$. The general time-dependent case is more difficult. Although some progress can be made by employing Fourier transforms, we have failed to find an analytic representation of the solution which is simple enough to be averaged explicitly. However, it is possible to solve the stochastic differential equation (1) numericall:. This work is described in Appendix $A$.

Now we proceed with a description of the bounding method. If we decompose $T$ into the ensemble average $\langle T\rangle(x)$ and the fluctuation $\delta T(x, t)$, then average Eq. (1) over the ensemble of $u$. we obtain a (locat) balance equation relating flux and dissipation.

$$
\frac{d}{d x}\langle\delta u \delta T\rangle-R^{-1} \frac{d^{2}}{d x^{2}}\langle T\rangle=0,
$$

where we assumed statistical stationarity. (Since $\langle u\rangle=0$, it is more suggestive and appropriate to write $\delta u$ instead of $u$.) Obviously, the continuity equation (2) is the first member of a hierarchy of moment equations which relate lower-order mornents to higher-order moments and exhibit the closure problem. It can be rewritten more usefully by integrating it once over $x$. Lpon using the boundary conditions, we obtain the following simpler relation between the flux $\Gamma(x, 0)$ and the (unknown) profile $\langle T\rangle(x)$ :

$$
\frac{d}{d x}\langle T\rangle(x)=R\left[\Gamma(x, 0)-\bar{\Gamma}(0)-R^{-1}\right]
$$

where

$$
\Gamma(x, \tau) \doteq\langle\delta u(t-\tau) \delta T(x, t)\rangle
$$

and $\bar{\Gamma}$ is the flux averaged over $x$ :

$$
\bar{\Gamma}(\tau) \doteq \int_{0}^{1} d x \Gamma(x, \tau) .
$$

By subtracting Eq. (2) from Eq. (1) we find an exact equation for the fluctuations:

$$
\begin{gathered}
\frac{\partial}{\partial t} \delta T(x, t)-\delta u(t) \frac{d}{d x}\langle T\rangle(x)-\delta u(t) \frac{\partial}{\partial x} \delta T(x, t)-\frac{\partial}{\partial x} \Gamma(x, 0)-R^{-1} \frac{\partial^{2}}{\partial x^{2}} \delta T(x, t)=0 . \\
-6-
\end{gathered}
$$


To obtain a variational functional, we begin by multiplying Eq. (4) by $\delta T(x . t)$ (at the same time- and space-point) and ensemble-averaging. We then obtain the "energr" balance equation

$$
\Gamma(x .0) \frac{d}{d x}\langle T\rangle-\frac{d}{d x}\left(\frac{1}{2} \delta u \delta T^{2}\right\rangle-R^{-1}\left\langle\delta T \frac{\partial^{2}}{\partial x^{2}} \delta T\right\rangle=0 .
$$

where "energy" is used generically for the scalar variance $\left.i \delta T^{2} / 2\right)$. The interpretations of the three terms in Eq. (5) as production, transfer, and dissipation, respectively. have been discussed by Krommes and Smith. Of course, Eq. (5): which relates the second-order moments to the third-order moment, is also part of a moment hierarchy: However, rather than attempting to close the hierarchy by approximating the triplet correlation. we proceed rigorously and annihilate it by integrating Eq. (5) over $x$ and using the rigid boundary conditions on $\delta T$. We obtain the equal-time constraint

$$
\bar{\Gamma}(0)=R \overline{\Delta \Gamma^{2}}+R^{-1} \overline{\left.\left(\frac{\partial \delta T}{\partial x}\right)^{2}\right)}
$$

where

$$
\lrcorner \Gamma(x) \doteq \Gamma(x, 0)-\bar{\Gamma}(0),
$$

bar means the integration over $x, E q$. (3) was used to eliminate the mean gradient, and the last term was obtained after integration by parts. Krommes and Smith called Eq. (6) the "basic" constraint.

It is easy to show that knowledge of $\bar{\Gamma}(0)$ determines the total flux of heat through the system. ${ }^{(t)}$ Thus one is led to the variational principle: Maximize $\bar{\Gamma}(0)$ uruder the constraint (6). This can be implemented most straight forwardly by introducing a Lagrange multiplier (for which we must solve self-consistently). Although this is not difficult for the reference model, it may be somewhat tedious in practice (especially in the generalizations to self-consistent situations). To attempt to circumvent this annovance, Krommes and Smith followed Howard ${ }^{(6)}$ and Busse ${ }^{(3)}$ in formulating an alternative, minimum, principle in which, under the basic constraint, the Lagrange multiplier does not appear explicitly. (It vanishes for the basic constraint.) Thus, following Krommes and Smith, it turns out that the basic variational principle is to

$$
\text { minimize } \mathcal{R}=\gamma \frac{R^{2} \overline{\Delta \Gamma^{2}}+\overline{\left((\partial \delta T / \partial x)^{2}\right)}}{\bar{\Gamma}(0)^{2}} \text { subject to } \bar{\Gamma}(0)=\gamma
$$

where $\mathcal{R}=R$ at the stationary point. The resulting function $\gamma(R)$ is the desired upper (basic) bound. The Euler-Lagrange equation which follows from Eq. ( 7 ) was solved in Ref. 4 [see Eq. (2.40)]. This result, independent of the Kubo number, is shown in Fig. 1 as the chain-dashed curves $\gamma_{b}$ for the three Reynolds numbers 5,10 , and 50 . The remaining information in this figure will be discussed below. 
Although the basic bound is quite rigorous. it is seriously flawed in general since it does not involve the Kubo number: due to the assumption of stationarity, the one-point constraint (6) involves no time dependence and cannot recognize two-time correlations. In other words. the basic bound behaves as though the Kubo number were infinite.

Clearly: to include the Kubo number in the expression of the upper bounds, one must consider two-time constraints, as was discussed by Krommes and Smith. They constructed a particular two-time constraint $C_{\mathrm{t}}(x, \tau)$ by multiplying Eq. (4) by $u\left(t^{\prime}\right)$ and ensembleaveraging to obtain

$$
0=C_{\mathrm{t}}(x, \tau) \doteq\left(\frac{\partial}{\partial \tau}-R^{-1} \frac{\partial^{2}}{\partial x^{2}}\right) \Gamma(x, \tau)-L^{r}(\tau) \frac{d}{d x}\langle T\rangle-\frac{\partial}{\partial x} \mathcal{T}(x, \tau)
$$

where $\tau$ is the time difference variable $\tau \doteq t-t^{\prime}, U$ is the specified autocorrelation function of $u-U(\tau) \doteq\left\langle\delta u(t) \delta u\left(t^{\prime}\right)\right\rangle=\exp (-|\tau| / K)$, and $T$ is a triplet cross-correlation function$T(x, \tau) \doteq\left\langle\delta u(t) \delta T(x, t) \delta u\left(t^{\prime}\right)\right\rangle$. Because this constraint is somewhat complicated, Krommes and Smith proceeded to obtain a reduced constraint $\bar{C}_{\mathrm{t}}=0$ by integrating over $x$ to annihilate the triplet cross-correlation. They then solved the resulting Euler-Lagrange equation and showed that the bound behaves reasonably for small kubo number-i.e., proportional to $K$. (In fact, for $K \rightarrow 0$ the bound reduces to the exact, quasilinear, result.) This bound is plotted in Fig. 1 as the solid curves $\gamma \mathrm{Ks}$. For finite $K$, the new bound is smaller than the basic bound, which is expected since more constraints were applied. (However, for $K \rightarrow \infty$ the two-time constraint asymptotes to the basic one; thus in that limit the new constraint is redundant and the bound is not improved.)

In fact, it is not necessary to annihilate the triplet cross-correlation in Eq- (8). A richer theory and a presumably better bound on the flux can be obtained by using the full Eq. (8) as the constraint, and this is what we shall explore here. Thus we minimize the functional

$$
\begin{aligned}
R=\gamma & \frac{R^{2} \overline{\Delta \Gamma^{2}}+\overline{\left\langle(\partial \delta T / \partial x)^{2}\right\rangle}}{\bar{\Gamma}(0)^{2}} \\
& +\int_{-\infty}^{\infty} d \tau \int_{0}^{1} d x \lambda_{0}(x, \tau) C_{\mathrm{t}}(x, \tau)+\int_{0}^{1} d x \lambda_{1}(x)(\delta T\rangle(x)+\lambda_{2}[\bar{\Gamma}(0)-\gamma]
\end{aligned}
$$

where we take into account the constraints $C_{\mathrm{t}}=0,\langle\delta T\rangle=0$, and $\bar{\Gamma}(0)=\gamma$ vio the Lagrange multipliers $\lambda_{0}, \lambda_{1}$, and $\lambda_{2}$. In the calculation of Krommes and Smith, $\lambda_{1}$ and $\lambda_{2}$ were omitted, as we will discuss momentarily. First, however, let us argue that the constraint $C_{\mathrm{t}}=0$ will. in fact, lead to an improvement in the bound, rather than to just a different bound. The issue is whether the new constraint consists of the old constraint $\bar{C}_{\mathrm{t}}=0$ plus an additional constraint-in which case the new bound cannot be worse than the old one-or whether the zew constraint is simply different-in which case there is no theorem 
about the relation of the new bound to the old one. To show that we are. in fact, adding a further constraint to the one of Krommes and Smith. we decompose $\lambda_{0}$ as

$$
\lambda_{0}(x, \tau)=\bar{\lambda}_{0}(\tau)-\Delta \lambda_{0}(x, \tau)
$$

where $\bar{\lambda}_{0}$ is the spatially averaged $\lambda_{0}$ and $\Delta \lambda_{0}$ is the deviation from $\bar{\lambda}_{0}$. Ising this, it is simple to rewrite the two-time constraint part of $\mathcal{R}$ as

$$
\int_{-\infty}^{\infty} d \tau \int_{0}^{1} d x \lambda_{0}(x, \tau) C_{t}(x, \tau)=\int_{-\infty}^{\infty} d \tau \bar{\lambda}_{0}(\tau) \bar{C}_{\mathrm{t}}(\tau)+\int_{-\infty}^{\infty} d \tau \int_{0}^{1} d x \Delta \lambda_{0}(x, \tau) C_{\mathrm{t}}(x, \tau)
$$

Clearly the first term on the right-hand side is nothing but the two-time-constraint part of the functional of Krommes and Smith. Since the $\Delta \lambda_{0}$ part represents another constraint additional to the old constraint of Krommes and Smith, we expect an improved bound. To ensure this, however, we must enforce the appropriate boundary conditions on $\Delta \lambda_{0}$. These can be determined by arguing that we should be able to recover the previous bound of Krommes and Smith as we let $\Delta \lambda_{0}$ vanish. Thus, in particular, we must require that $\Delta \lambda_{0}$ vanishes on the boundaries:

$$
\Delta \lambda_{0}(0, \tau)=\Delta \lambda_{0}(1, r)=0
$$

In the present calculation, we must explicitly enforce the constraint $\langle\delta T\rangle=0$ for the following reason. Since $C_{\mathrm{t}}$ has a cubic nonlinearity, the resulting Euler-Lagrange equation is quadratically nonlinear. Hence, in general the ensemble average of the Euler-Lagrange equation does not automatically vanish. (Krommes and Smith annihilated the cubic term, so their Euler-Lagrange equation was linear and the constraint $\langle\delta T\rangle=0$ was automatic; for them, $\lambda_{1} \equiv 0$.)

The term in $\lambda_{2}$ is required to ensure that the Euler-Lagrange equation satisfies the one-time constraint (6). This is not satisfied automatically since the two-time constraint $C_{\mathrm{t}}$ involves a constant term [when the gradient of the profile is eliminated via Eq. (3)], and this constant disappears under the functional derivative required to generate the EulerLagrange equation. This is a property of constraints such as $C_{2}$ for the basic bound $\lambda_{2}=0$ because the basic functional is homogeneous of degree 0 in $\delta T$. This is why Busse, Krommes and Smith, and others did not bother to introduce $\lambda_{2}$ explicitly in their calculations of the basic bound. The situation is different in the two-time calculation of Krommes and Smith, where they should have included this constraint. Fortunately, their final result stands. In their calculation $\bar{\lambda}_{0}$ was directly related to $d \overrightarrow{\mathrm{T}} / d \tau$. When one includes $\lambda_{2}$, it can be shown that $\lambda_{2}$ combines with $\bar{\lambda}_{0}$ to give $d \vec{\Gamma} / d \tau$. Thus the new $\bar{\lambda}_{0}$ differs from that of Kiommes and Smith by a constant in space; however, in terms of the new $\bar{\lambda}_{0}$, the equations for the bounding flux are the same in both calculations. 
Upon taking the functional derivative of Eq. (9) with respect to $\delta T$ and using Eq. (3). we are led to the Euler-Lagrange equation

$$
\begin{aligned}
0=-R^{-1} & \frac{\partial^{2}}{\partial x^{2}} \delta T(x, t)+\delta u(t) \frac{d}{d x}(T)-\frac{\partial}{\partial t} \int_{-\infty}^{\infty} d \bar{\tau} \Lambda_{0}(x, \bar{\tau}) \delta u(t-\bar{\tau}) \\
-R^{-1} & \frac{\partial^{2}}{\partial x^{2}} \int_{-\infty}^{\infty} d \bar{\tau} \Lambda_{0}(x, \bar{\tau}) \delta u(t-\bar{\tau})+R \int_{-\infty}^{\infty} d \bar{\tau} \Lambda_{0}(x, \bar{\tau}) U(\bar{\tau}) \delta u(t) \\
& -R \int_{-\infty}^{\infty} d \bar{\tau} \int_{0}^{l} d \bar{x} \Lambda_{0}(\bar{x}, \bar{\tau}) U(\bar{\tau}) \delta u(t)-\frac{\partial}{\partial x} \int_{-\infty}^{\infty} d \bar{\tau} \Lambda_{0}(x, \bar{\tau}) \delta u(t-\bar{\tau}) \delta u(t) \\
& -\Lambda_{1}(x)-\Lambda_{2} \delta u(t),
\end{aligned}
$$

where

$$
\Lambda_{i} \doteq\left(\frac{\gamma}{2 R}\right) \lambda_{i}
$$

This result omits various Dirac delta functions concentrated at the boundaries, because we have chosen to define $\delta T(x, t)$ only on the open interval $(0,1)$. For more discussion on this point, we refer the reader to Appendix $B$.

It is useful to proceed by eliminating the subsidiary multipliers $\Lambda_{1}$ and $A_{2}$ in terms of $\Lambda_{0}$. This can be done by requiring that the associated constraints be satisfied. First, by ensemble-averaging. Eq. (11), we can determine $\Lambda_{1}$ in terms of $\lambda_{0}$ :

$$
\Lambda_{1}(x)=\frac{\partial}{\partial x} \int_{-\infty}^{\infty} d \bar{\tau} \Lambda_{0}(x, \bar{\tau}) U(\bar{\tau})
$$

(Note that $\Lambda_{1}$ vanishes when $\Lambda_{0}$ is independent of $x$, which is the case for the reduced constraint $\bar{C}_{\mathrm{t}}$ of Krommes and Smith.) Similarly, to find $\Lambda_{2}$ in terms of $\Lambda_{0}$, we multiply Eq. (11) by $\delta T(x, t)$ (again, at equal space- and time-points), ensemble-average, and integrate over $x$. Upon using the one-time constraint, we obtain

$$
\Lambda_{2}=-\gamma^{-1} \int_{-\infty}^{\infty} d \bar{\tau} \int_{0}^{1} d \bar{x} \Lambda_{0}(\bar{x}, \bar{\tau}) U(\bar{\tau})
$$

(Note that $\Lambda_{2}$ does not vanish even when $\Lambda_{0}$ is independent of $x_{\cdot}$ ) Upon inserting $\Lambda_{1}$ and $\Lambda_{2}$ into Eq. (11), one can write the Euler-Lagrange equation much more symmetrically as

$$
\begin{gathered}
0=-R^{-1} \frac{\partial^{2}}{\partial x^{2}} \delta T(x, t)+\delta u(t) \frac{d}{d x}\langle T\rangle-\frac{\partial}{\partial t} \int_{-\infty}^{\infty} d \bar{\tau} \Lambda_{0}(x, \bar{\tau}) \delta u(t-\bar{\tau}) \\
-R^{-1} \frac{\partial^{2}}{\partial x^{2}} \int_{-\infty}^{\infty} d \bar{\tau} \Lambda_{0}(x, \bar{\tau}) \delta u(t-\bar{\tau})-\frac{\partial}{\partial x} \int_{-\infty}^{\infty} d \bar{\tau} \Lambda_{0}(x, \bar{\tau})[\delta u(t-\bar{\tau}) \delta u(t)-U(\bar{\tau})] \\
+R \int_{-\infty}^{\infty} d \bar{\tau} \Lambda_{0}(x, \bar{\tau}) U(\bar{\tau}) \delta u(t) \\
-R\left[1+(R \gamma)^{-1}\right] \int_{-\infty}^{\infty} d \bar{\tau} \int_{0}^{1} d \bar{x} \Lambda_{0}(\bar{x}, \bar{\tau}) U(\bar{\tau}) \delta u(t) \\
-10-
\end{gathered}
$$


The remaining task is to find the undetermined Lagrange multiplier $\mathrm{A}_{0}$ self-consistently and then solve for the flux. To do this we follow the general procedure of Krommes and Snith. who pointed out that it is unnecessary to actually solve for $\delta T$ itself. Rather. one can directly construct an equation for $\Gamma$ by multiplying the Euler-Lagrange equation by $\delta u\left(t^{\prime}\right)$ and ensemble-averaging, using the Gaussian property of $\delta u(t)$. We obtain

$$
\begin{aligned}
0=- & R^{-1} \frac{\partial^{2}}{\partial x^{2}} \Gamma(x, \tau)+R L(\tau)\left[\Gamma(x, 0)-\bar{\Gamma}(0)-R^{-1}\right] \\
& -\left(\frac{\partial}{\partial \tau}-R^{-1} \frac{\partial^{2}}{\partial x^{2}}\right) \psi(x, \tau)-R U(\tau) i \psi(x, 0)-\bar{\psi}(0)-(R \gamma)^{-1} \bar{\psi}(0)
\end{aligned}
$$

where $\psi$ is the convolution of $\Lambda_{0}$ and $U$ :

$$
\dot{\psi}(x, \tau) \doteq \int_{-\infty}^{\infty} i \bar{\tau} \Lambda_{0}(x, \tilde{\tau}) U(\tau-\dot{\tau})
$$

Because the boundary conditions (10) imply

$$
\Delta \psi(0, \tau)=\Delta \psi(1, \tau)=0,
$$

where

$$
\Delta \psi(x, \tau) \doteq \psi(x, \tau)-\bar{\psi}(\tau)
$$

it is more convenient to express Eq. (14) in terms of $\Delta \psi$ rather than $\psi$ itself. To do this, we integrate Eq. (14) and $C_{\mathrm{t}}$ over $\boldsymbol{x}$ and combine the results to obtain

$$
\left.\frac{d}{d \tau} \bar{\psi}(\tau)+\gamma^{-1} \bar{\psi}(0) U(\tau)=-\frac{d}{d \tau} \bar{\Gamma}(\tau)-R^{-1} \Delta \psi^{\prime}(0)\right]_{0}^{1} U(\tau)
$$

We may now use this result to eliminate $\bar{\psi}$ in favor of $\Delta \psi$ in $\mathrm{Eq}$. (14); we finally obtain

$$
\begin{aligned}
0=\frac{d}{d \tau} & \bar{\Gamma}(\tau)-R^{-1} \frac{\partial^{2}}{\partial x^{2}} \Gamma(x, \tau)+R U(\tau)\left[\Gamma(x, 0)-\bar{\Gamma}(0)-R^{-1}\right] \\
& -\left(\frac{\partial}{\partial \tau}+R^{-1} \frac{\partial^{2}}{\partial x^{2}}\right) \Delta \psi(x, \tau)+R U(\tau) \Delta \psi(x, 0)-2 R^{-1} \Delta \psi^{\prime}(0) U(\tau) .
\end{aligned}
$$

In obtaining the last term of $\mathrm{Eq} .(16)$, we used the symmetries

$$
\Gamma(x, \tau)=\Gamma(1-x, \tau), \quad \Delta \psi(x, \tau)=\Delta \psi(1-x, \tau)
$$

to deduce

$$
\Delta \psi^{\prime}(0)=-\Delta \psi^{\prime}(1)
$$

Note that for $\Delta \psi=0 \mathrm{Eq}$. (16) reduces properly to Eq. (5.17) of Krommes and Smith. 
To obtain a closed set for $\Gamma$ and $v$. we need one more equation. This may be found br multipltying Eq. (13) by $\delta u(t) \hat{c} u(t-\tau)$ and ensemble-averaging. We obtain

$$
-R^{-1} \frac{\partial^{2}}{\partial x^{2}} T(x, \pi)-\frac{\partial}{\partial x} v(x, \tau)-v(x, 0) U(\tau)=0 .
$$

where we used

$$
\delta u(t) \tilde{\partial} u(t-\tau) \delta u(t-\bar{\tau}) \delta u(t))-C(\tau) C(\tau)=\ell(\tau-\bar{\tau})-C(\tau) \zeta(\bar{\tau}) .
$$

After we integrate once over $x$, we can rewrite Eq. (17) in a form appropriaze for use in the two-time constraint (B):

$$
\frac{\partial}{\partial x} T(x, \tau)=-R . \Delta v(x . \tau)-\Delta w(x, 0)(\tau) !
$$

Equations (8), (16), and (18) form a closed set of equations for $\Gamma, \Delta \psi$, and $T$.

If we take

$$
U(T)=\exp (-|\tau| / K)
$$

for the autocorrelation function. we can simplify the problem by invoking separation of variables,

$$
\Gamma(x, \tau)=\Gamma(x) U(\tau), \quad \Delta \underline{\psi}(x, \tau)=\Delta \Psi(x) \mathcal{L}(\tau) .
$$

In this case the Lagrange multiplier behaves in time as

$$
\Delta \Lambda_{0}(x, T)=\Delta \Psi(x) \delta(\tau)
$$

as can be determined by solving Eq. (15) by Fourier-transforming in time. Using the expression (18) for the triplet cross-correlation together with Eqs. (19), we can reduce the constraint (8) to

$$
-R^{-2} \frac{d^{2}}{d x^{2}} \Gamma \div\left[1+(K R)^{-1}\right] \Gamma-\left(\gamma-R^{-1}\right)-2 \Delta \Psi=0
$$

To get the above we take $U(\tau)=\exp (\tau / K)$, since because of the diffusion operator the system evolves in the forwand direction in time and we are interested in the fux at $T=0$ which evolves from the state at $\tau=-\infty$. In the same manner we reduce Eq. (16) to

$$
\begin{aligned}
0=- & R^{-2} \frac{d^{2}}{d x^{2}} \Gamma \div \Gamma-\gamma\left[1-(K R)^{-i}+(R y)^{-1}\right] \\
& -R^{-2} \frac{d^{2}}{d x^{2}} \Delta \Psi+\left[1-(K R)^{-1}\right] \Delta \Psi-2 R^{-2} \Delta \Psi^{\prime}(0) .
\end{aligned}
$$

In the case when $K \rightarrow \infty$, one can eliminate the first two terms on the right-hand side of Eq- (21), by using Eq- (20), to arrive at an equation which involves only $\Delta \Psi$. One 
can then argue that $\Delta \Psi=0$. since the eouation is homogeneous in $\lambda \Psi$ and $\Delta \Psi=0$ also satisfies the boundary conditions. Since the triplet cross-correlation also vanishes. we reproduce the basic result of hrommes and Smith in this case. The reason for this is that when $\boldsymbol{k} \rightarrow \infty$, the two-time constraint reduces to the one-time constraint: The time-derivative term of Eq. (8) vanishes and the solution $\delta T$ following the variational principle ( $\overline{7}$ ) satisfies Eq. (8).

The case of finite $K$ is more involved. After straightforward algebra, one can combine Eqs. (20) and (21) to obtain

$$
\begin{aligned}
& R^{-4} \frac{d^{4}}{d x^{4}} \Gamma-4 R^{-2} \frac{d^{2}}{d x^{2}} \Gamma-\left[3-(K R)^{-2}\right] \Gamma \\
& \left.\quad=\left\{3 \gamma\left[1-(K R)^{-1}\right]-R^{-1}\left[3-(K R)^{-1}\right]\right\}-4 R^{-2}\right\rfloor \Psi^{\prime}(0),
\end{aligned}
$$

with boundary conditions

$$
\begin{aligned}
\Gamma(x)_{2=0,1} & =0 \\
R^{-2} \Gamma^{\prime \prime}(x)_{z=0,1} & =-\left(\gamma-R^{-1}\right) .
\end{aligned}
$$

The boundary conditions on $\Gamma^{\prime \prime}$ follow by applying Eq. (10) to Eq. (20).j We can solve the above analytically: first, solve for $\Gamma$ as a function of $\gamma$ and $\Delta \Psi^{\prime}(0)$; next, use this result in Eq. (20) to obtain $\Delta \Psi^{\prime}(0)$; finally, integrate $\Gamma$ over $x$ in order to obtain an equation for $\gamma$. Since the final expression for $\gamma$ is long and messy; we do not write it here. However, numerical display of the analytic solution is straightforward and is shown in Fig. 1 as the dashed curves $\gamma_{t}$. Both this and the two-time bound of Krommes and Smith behave properly for the important limiting cases: $\gamma \sim K$ in the quasilinear regime; $\gamma=O(1)$ in the strong turbulence regime. It is evident that our present calculations have improved the bound, although insignificantly. That $\gamma_{K s}$ and $\gamma_{k}$ should be qualitatively similar is not surprising, since the two two-time bounds are equal at the two end points $K=0$ and $K=\infty$.

The only way to improve the situation at $K=\infty$ is to consider a constraint which embraces more equal-time effects, and it is natural to consider fourth-order effects. A new constraint for that purpose is obtained by multiplying Eq. (4) by $\delta u^{2}\left(t^{\prime}\right)$ and ensembleaveraging:

$$
\begin{aligned}
C_{f}(x, \tau) & =\left(\frac{\partial}{\partial \tau}-R^{-1} \frac{\partial^{2}}{\partial x^{2}}\right)\left\langle\delta u^{2}\left(t^{\prime}\right) \delta T(x, t)\right\rangle-\frac{d}{d x} \Gamma(x, 0)+\frac{\partial}{\partial x}\left\langle\delta u^{2}\left(t^{\prime}\right) \delta u(t) \delta T(x, t)\right) \\
& =0
\end{aligned}
$$


The corresponding variational principle is

$$
\begin{aligned}
\operatorname{minimize} \mathcal{R}= & \frac{R^{2} \overline{\Delta \Gamma^{2}}-\overline{\left\langle(\partial \delta T / \partial x)^{2}\right.}}{\bar{\Gamma}(0)^{2}}-\int_{-\infty}^{\infty} d \tau \hat{\lambda}_{0}(\tau) \bar{C}_{\mathrm{t}}(\tau) \\
& -\int_{-\infty}^{\infty} d \tau \int_{0}^{1} d x \hat{\lambda}_{1}(x . \tau) C_{\mathrm{f}}(x . \tau)-\int_{0}^{1} d x \hat{\lambda}_{2}(x)_{i} \delta T \\
& -\bar{\lambda}_{3} \bar{\Gamma}(0)-\tau
\end{aligned}
$$

Notice that we are using the constraint $\bar{C}_{t} ;$ we are not including the triplet correlational effect in the two-time constraint Eq. (8), which we considered earlier. Some constraint such as $\bar{C}_{\mathrm{t}}=0$ is required in order to ensure that the Kubo number scaling in the limit $K \rightarrow 0$ is captured correctly. In the absence of $\bar{C}_{t}$, the resulting bound does not reduce to quasilinear theory as $K \rightarrow 0$.

From Eq. (24), the Euler-Lagrange equation follows as

$$
\begin{aligned}
0=- & R^{-1} \frac{\partial^{2}}{\partial x^{2}} \delta T(x, t)-\delta u(t) \frac{d}{d x}\langle T\rangle-\int_{-\infty}^{\infty} d \bar{\tau} \hat{\Lambda}_{0}(\bar{\tau})\left[\frac{\partial}{\partial t} \delta u(t-\bar{\tau})+\gamma^{-1} C^{\prime}(\bar{\tau}) \delta u(t)\right] \\
& -\left(\frac{\partial}{\partial t}-R^{-1} \frac{\partial^{2}}{\partial x^{2}}+\delta u(t) \frac{\partial}{\partial x}\right) \int_{-\infty}^{\infty} d \bar{\tau} \hat{\Lambda}_{1}(x, \bar{\tau})\left[\delta u^{2}(t-\bar{\tau})-1\right],
\end{aligned}
$$

where $\hat{\lambda}_{2}$ and $\hat{\lambda}_{3}$ are determined in terms of $\hat{\lambda}_{0}$ and $\hat{\lambda}_{1}$ as before,

$$
\begin{aligned}
& \hat{\Lambda}_{2}=R^{-1} \frac{\partial^{2}}{\partial x^{2}} \int_{-\infty}^{\infty} d \overline{\bar{\tau}} \hat{\Lambda}_{1}(x, \bar{\tau}), \\
& \hat{\hat{\Lambda}}_{3}=-\gamma^{-1} \int_{-\infty}^{\infty} d \bar{\tau} \hat{\Lambda}_{0}(\bar{\tau}) U(\bar{\tau}) .
\end{aligned}
$$

The boundary conditions for $\widehat{\Lambda}_{1}$ are $\widehat{\Lambda}_{1}=0$ at $x=0$ and $x=1$. Since $\delta u$ is independent of $\delta T$, higher-order cross-correlations can be expressed in terms of the flux $\Gamma$ by maltiplying the Euler-Lagrange equation by the corresponding number of $\delta u$ 's,

$$
\begin{aligned}
& 0=-R^{-1} \frac{\partial^{2}}{\partial x^{2}} \Gamma(x, \tau)+R U(\tau)\left[\Gamma(x, 0)-\bar{\Gamma}(0)-R^{-1}\right] \\
& -\int_{-\infty}^{\infty} d \bar{\tau} \bar{\Lambda}_{0}(\bar{\tau})\left[\frac{\partial}{\partial \tau} U(\tau-\bar{\tau})+\gamma^{-1} U(\bar{\tau}) U(\tau)\right] \\
& -2 \frac{\partial}{\partial x} \int_{-\infty}^{\infty} d \bar{\tau} \hat{\Lambda}_{1}(x, \bar{\tau}) U(\bar{\tau}) U(\tau-\bar{\tau}), \\
& 0=-R^{-1} \frac{\partial^{2}}{\partial x^{2}}\left\langle\delta u^{2}\left(t^{\prime}\right) \delta T(x, t)\right\rangle \\
& -\left(\frac{\partial}{\partial \tau}+R^{-1} \frac{\partial^{2}}{\partial x^{2}}\right) \int_{-\infty}^{\infty} d \bar{\tau} \hat{\Lambda}_{1}(x, \bar{\tau})\left(\delta u^{2}\left(t^{\prime}\right) \delta u^{2}(t-\bar{\tau})\right\rangle,
\end{aligned}
$$




$$
\begin{aligned}
& 0=-R^{-1} \frac{\partial^{2}}{\partial x^{2}}\left\langle\delta u^{2}\left(t^{\prime}\right) \delta u\left(t^{\prime \prime}\right) \delta T(x, t)\right\rangle-R\left\langle\delta u^{2}\left(t^{\prime}\right) \delta u\left(t^{\prime \prime}\right) \delta u(t)\right)\left[\Gamma(x, 0)-\bar{\Gamma}(0)-R^{-1}\right. \\
& -\int_{-\infty}^{\infty} d \bar{\tau} \widehat{\Lambda}_{0}(\bar{\tau})\left[\frac{\partial}{\partial t}\left\langle\delta u^{2}\left(t^{\prime}\right) \delta u\left(\iota^{\prime \prime}\right) \delta u(t-\bar{\tau})\right\rangle-\gamma^{-1} U(\bar{\tau})\left\langle\delta u^{2}\left(t^{\prime}\right) \delta u\left(t^{\prime \prime}\right) \delta u(t)\right\rangle\right] \\
& -\frac{\partial}{\partial x} \int_{-\infty}^{\infty} d \bar{\tau} \hat{\Lambda}_{1}(x, \bar{\tau}) \\
& \times\left(\delta u^{2}\left(t^{\prime}\right) \delta u\left(t^{\prime \prime}\right) \delta u(t) \delta u^{2}(t-\bar{\tau})\right\rangle-\left\langle\delta u^{2}\left(t^{\prime}\right) \delta u\left(t^{\prime \prime}\right) \delta u(t)\right\rangle^{-} .
\end{aligned}
$$

First of all, by integrating Eq. (26) over $x$ and using the constraint $\bar{C}_{\mathrm{t}}=0$, we find that $\overline{\mathrm{T}}_{0}$ is the same as that of Krommes and Smith. Also, assurning the temporal behavior of the Lagrange multipliers to be the Dirac deita function and separating variables, we end up with two equations, (26) and (23), where we have eliminated the triplet and fourthorder cross-correlations in favor of the flux $\Gamma$ and the Lagrange multiplier $\widehat{\Lambda}_{1}$ by using Eqs. (27) and (28). An easy way to solve these coupled equations is to first solve Eq. (26) for $\Gamma$, then to put that expression into Eq. (23) to obtain a sixth-order, constant-coefficient ordinary differential equation for $\Gamma$, where the extra orders are due to the fact that we differentiate Eqs. (26) and (23) to obtain the final differential equation. The general solution of this equation has six constants. In order to determine these constants uniquely we solve for $\widehat{\lambda}_{1}$, the triplet cross-correlation, and the fourth-order cross-correlation in terms of the constants. Then we use the boundary conditions and compare the coefficients of $x^{2}, x$, and the constant terms of Eq. (23).

The final result is plotted in Fig. 1 as the dotted curves $\gamma_{f}$, and is seen to be significantly better than the previous ones. Also shown by the solid circles with error bars are representative points from the "exact" rumerical solution of Eq. (1), described in Appendix $A$, for $R=10$. (It is an accident that these points happen to lie close to the bound $k$ s for $R=5$.) The exact results for $K=\infty$ follow from Eq. (2.14) of Krommes and Smith and are indicared by the arrows on the right-hand ordinate. (The eye may perceive a discontinuity between the exact points at $K=5$ and $K=\infty$, but this is an illusion caused by moving the point at $K=\infty$ in to $K=6$; compare the similar situation for $\gamma_{\mathrm{b}}$ and $\gamma_{\mathrm{KS}}$ at $\boldsymbol{R}=50$.) Clearly the newest bound leaves little room for improvement.

Although in the present case we are able to obtain the exact solution, the difficulty of that procedure actually provides a compelling argument for the bounding method. As we describe in Appendix $A$, it is difficult to obtain a precise value for the flux due to problems with statistical noise. Although the noise can always be reduced by using more realizations, the computations then become very long and expensive, even for the very simple, onedimensional reference model. On the other hand, even our most complicated bound can be obtained readily and precisely by quite straightforward analytic and numerical analysis. Although it will not always be the case that the bounding method will be superior, it appears to be a viable option in a variety of problems similar to the present one. 


\section{BOUNDS ON PARTICLE TRANSPORT IN A TURBULENT PLASMA DUE TO STOCHASTIC MAGNETIC FIELDS}

In the previous section. we dealt with a ststem where the correlation length $L_{c}$ associated with the advecting velocity field was infinite. To be more realistic, we need to consider the case where the correlation length is finite. Because certain shortcuts are possible in the case of infinite $L_{c}$, it is not immediately clear how manageable the optimum theory will be in the more general situation. A specific example is the problem of particle transport due to specified stochastic magnetic fields, and we discuss that in this section. This problem was also considered by Krommes and Smith from the point of view of the optimum theory; however. they only considered the basic bound.

Since the field lines are stochastic, particles stuck to the field lines also move in a random fashion and there results a transport of particles, energy, and so forth across the field lines. ${ }^{(\theta)}$ However, the precise nature of this transport is subtle and depends critically an the collisionality of the particles. If the particles'undergo no collisions at all, their diffusion coefficient is related straightforwardly to that of the magnet ic lines, and is called the collisionless result. ${ }^{(15)}$ A more interesting situation arises when the particles are allowed to diffuse along the actual lines, but are never scattered by perpendicular diffusion from their original lines. Then, the "double diffusion, ${ }^{n(11)}$ of the particles along the lines and of the lines themselves, leads to a transport process slower than diffusive, which means that the diffusion coefficient vanishes. An even very slight amount of perpendicular diffusion, however, is sufficient ${ }^{(10,11)}$ to restore a radial diffusion process for the particles, and in a wide parameter range of relevance to many experiments the "collisionless" result is again recovered. When the parallel collisionality becomes sufficiently large, other regimes and results are appropriate and Krommes, Oberman, and Kleva(11) have shown how these regimes are continuously connected in parameter space. Krommes and Smith considered bounds for arbitrary collisionality, ${ }^{(4)}$ but since they considered only the basic bound they were unable to obtain the collisionless scaling in the quasilinear regime of short $L_{c}$, which is of considerable experimental relevance.

To illustrate the optimum techniques for finite $L_{c}$, we shall consider a model that is most appropriate for the collisionless, quasilinear regime. Specifically, we consider the model equation ${ }^{(1)}$

$$
\frac{\partial f}{\partial t}+v \frac{\partial f}{\partial \ell}+\delta u \cdot \nabla f-R^{-1} \nabla_{\perp}^{2} f=0
$$

where $\nabla_{\perp}^{2} \doteq \partial^{2} / \partial x^{2}+\partial^{2} / \partial y^{2}, \ell$ is the arc length along the unperturbed field line, $\partial / \partial \ell \doteq$ $\partial / \partial z+\left(x / L_{s}\right) \partial / \partial y$ ( $L_{s}$ is the shear length), $R \doteq \bar{u} L / \kappa_{e}$ is the Reynolds number, and $\delta \boldsymbol{u}$ describes the stochastic field, $\delta \boldsymbol{u} \doteq v \delta b \equiv \hat{z} \delta u_{x}+\ddot{y} \delta u_{y}$. Here $\delta b$ is the dimensionless magnetic field fluctuation from an equilibrium configuration with good flux surfaces. We have normalized the density to $n_{0}$, the perpendicular spatial coordinates $x$ and $y$ to $L$, $z$ to $L / \bar{b}$, where $\bar{b}=\left(\delta b_{z}^{2}\right)^{1 / 2}$, the parallel velocity $v$ to the thermal velocity $v_{t}$, the time $t$ to $L / \bar{u}$, where $\bar{u}=\bar{b} v_{\mathrm{t}}$, the particle distribution function $f$ to the Maxwellian $f_{M}$, and the magnetic field to the average uniform field strength $B_{0}$ in the $z$ direction. In Eq. (29), the 
second term represents the effect of particles streaming along the unperturbed field lines and the third term describes the advection of particles due to the stochastic field lines. The last term describes the effects of dissiparion. which we may think of (11) as modelling either real classical collisional diffusion or possibly the effect of the $\boldsymbol{E} \times \boldsymbol{B}$ nonlinearities in real space. Note. in particular, that we have completely omitted the effects of collisions on the parallel velocity. In the limit of infinite Reynolds number, the model correctly describes the completely collistonless (and nonphysical) situation, in which the particles never leave their original lines. In general, however. since the collision operator is absent the model does not correspond precisely to any of the physical colljsionality regimes described above, so some of the transport regimes predicted by the model are nonphysical. This is not of concern. though, since our interests here are pedagogical. The bound we shall obtain in the limit of small $L_{c}$ and $R \rightarrow \infty$ is valid for the important collisionless situation in which both the parallel and perpendicular collisionality are small, although nonzero. Thus, although the model does not completely capture the physics of a realistic experimental situation, it is rich enough to display important features and difficulties of the optimum procedure applied to passive situations with a finite autocorrelation length. There is no difficulty in principle in including the effects of parallel collisionality: see the work of Krommes and Smith.

As usual, the optimum question is: Determine bounds on the flux through a bounded region, under various rjgorous constraints. Since we work in the collisionless situation, it is sufficient to determine the particle flux $\Gamma_{v}$ as a function of velocity; various physical fluxes are appropriate velocity moments of $\Gamma_{v}$.

The nature of the boundary conditions we shall impose requires some discussion. Specifically, we concentrate on a region of overlapping magnetic islands, bounded by flux (KAM) surfaces which we take for simplicity of description to be planes lying at $x=x_{a}$ and $x=x_{b}$. Effectively, our model calculation, in which the advecting field does not vanish at $x=0$ or $x=L$, corresponds to working in a subregion $[0, L]$ such that $x_{a}<0 \leq x \leq$ $L<x_{b}$; this can be thought of as an "interior slice" of a larger stochastic system. We shall impose the boundary conditions

$$
n(0)=n_{0}, \quad n(L)=0
$$

However, for such a slice such rigid boundary conditions are inappropriate, and one is then led to question just how seriously one can take the resulting bounds. Fortunately, we can appeal to the discussion of Krommes and Smith, who argue that in the limit $L_{\mathrm{c}} \ll L$ the effects of the boundary conditions are localized near the edges of the slice and negligibly affect the global transport.

In the following, we shall exploit an important physical assumption about the perturbed magnetic field-namely, that it is perpendicular to the unperturbed $B_{z}$ :

$$
\begin{gathered}
\delta b_{z}=0 . \\
-17-
\end{gathered}
$$


This approximation assumes that the field compression $\delta\left(B^{2}\right)$ is negligible and. in a theory of microinstabilities, corresponds to ignoring the compressional Alfvén wave.

The structure of Eq. (29) is quite similar to that of the previous example except that here we have a three-dimensional problem and the "advecting velocity field" $\delta u$ depends on space as well as on time. Thus the procedure we will follow is basically the same as in Sec. 2 except that here we will neglect the triplei and nigher-order correlations: First, we find the equal-time, equal-space-i.e., one-point-constraint from the "energy" balance. However, as before, this basic constraint does not describe the effects of either short autocorrelation time or length, which are important in physical situations. Thus we add a two-point constraint to complete the variational functional. Then, we proceed in a straightforward way to obtain the Euler-Lagrange equation. The detailed calculation is described in Appendix $C$; the final equation for the bounding flux is

$$
\begin{aligned}
0=- & R^{-1} \nabla_{\perp}^{2} \Gamma_{v}\left(x, z^{\prime}, \bar{y}, \bar{z}, \tau\right)+R\left[\Gamma_{v}(x, x, 0)-\bar{\Gamma}_{y}(0)-R^{-1} !\right. \\
& +\alpha U\left(x, x^{\prime}, \bar{y}, \bar{z}, \tau\right),
\end{aligned}
$$

where $\Gamma_{v}\left(x, z^{\prime}, \bar{y}, \bar{z}, \tau\right)$ is the two-point particle flux as a function of velocity in the $x$ djrection, $\bar{\Gamma}_{v}(0)$ is the one-point flux integrated over $x_{1} U$ is the autocorrelation function of $\delta u_{x}$, and

$$
\alpha \doteq\left[\int_{-\infty}^{\infty} d \bar{y} \overline{\bar{U}}(\bar{y}, \bar{z}, \tau)\right]^{-1}\left[\left(\frac{\partial}{\partial \tau}+v \frac{\partial}{\partial \bar{z}}\right) \int_{-\infty}^{\infty} d \bar{y} \overline{\bar{\Gamma}}_{v}(\bar{y}, \tilde{z}, \tau)\right]
$$

Also, the double bar represents integration over both $x$ and $x^{\prime}$, and 0 stands for $(\bar{y}, \bar{z}, \tau)=$ $(0,0,0)$. An interesting feature of this equation is that $x^{\prime}$ enters the problem as a parameter since there is no differential operator with respect to $x^{\prime}$ in the equation; nevertheless, it is still an independent variable and the flux $\Gamma_{\mathrm{v}}$ in the second term is computed at the point $x^{\prime}=x$. Also, notice that the coefficient $\alpha$ of $U$ in the last term is a constant. [See Eq. (50).]

We can simplify the problem by choosing the correlation function to be separable and of Markovian type:

$$
U\left(x, x^{\prime}, \bar{y}, \bar{z}, \tau\right)=v^{2} \exp \left(-\left|x-x^{\prime}\right| / l_{z}\right) \exp \left(-|\bar{y}| / l_{y}\right) \exp \left(-|\bar{z}| / l_{z}\right) \exp (-|\tau| / K)
$$

where the l's and $K$ are the correlation lengths and correlation time, respectively. By employing separation of variables, we obtain

$$
\begin{gathered}
0=-R^{-2}\left(\frac{\partial^{2}}{\partial x^{2}}+l_{y}^{-2}\right) \Gamma_{v}\left(x, x^{\prime}\right)+v^{2}\left[\Gamma_{v}(x, x)-\bar{\Gamma}_{y}(0)-R^{-1}\right] \exp \left(-\left|x-x^{\prime}\right| / l_{z}\right) \\
+R^{-1}\left(K^{-1}+|v| l_{z}^{-1}\right) \frac{\left[\int_{0}^{1} d \bar{x} d \bar{x}^{\prime} \Gamma_{v}\left(\bar{x}, \bar{x}^{\prime}\right)\right]}{\left[\int_{0}^{1} d \bar{x} d \bar{x}^{\prime} \exp \left(-\left|\bar{x}-\bar{x}^{\prime}\right| / l_{x}\right)\right]} \exp \left(-\left|x-x^{\prime}\right| / l_{x}\right) . \\
-18-
\end{gathered}
$$


In general, we can obtain a solution $\bar{\Gamma}_{0}(0)$ parametrized by $l_{x}, l_{y}, l_{z}, K$, and $R$.

For the extreme case that the perpendicular correlation lengths $l_{z}$ and $l_{y}$ are very long, Eg. (31) reduces to Eq. (5.19) of Krommes and Smith except for the factor of $v^{2}$ and the streaming effect. The dimensionless bound $\gamma$ is found to be

$$
\gamma^{-1}=\gamma_{\infty}^{-1}-\left(v^{2} K\right)^{-1}-\left(i v L_{z}\right)^{-1}
$$

where $\gamma_{\infty}$ is the basic bound ${ }^{(-)}$for infinite $K$ and $l_{z}$ :

$$
\gamma_{\infty}=R^{-1}\left[I-\frac{2}{R v} \tanh \left(\frac{R v}{2}\right)\right] /\left[\frac{2}{R v} \tanh \left(\frac{R v}{2}\right)\right] .
$$

For the quasilinear regime $R \gg 1, K \ll 1$, and $l_{z} \ll 1$, the dimensionless bound behaves as

$$
\gamma \sim \min \left(v^{2} K, v l_{z}\right)
$$

or. after restoring the dimensions,

$$
\bar{\Gamma}_{v} \sim \bar{b}^{2}\left(\frac{v^{2}}{L}\right) \min \left(\tau_{\mathrm{ac}}, l_{z} / v \mid\right)
$$

In the static limit, this reproduces the "Rechester-Rosenbluth" estimate of the diffusion coefficient $D$ in Refs. 10,16 , and 11 ,

$$
D \sim \bar{b}^{2} l_{z}|v|
$$

and in turn the magnetic diffusion coefficient $D_{\mathrm{m}}$ :

$$
D_{\mathrm{m}} \sim \bar{b}^{2} l_{z}
$$

Also, it agrees with the strong turbulence scaling ${ }^{(4)}$

$$
D_{\mathrm{m}} \sim \bar{b} L
$$

when $R \gg 1$ and $l_{2} \gg 1$.

The extreme limit where both $l_{x}$ and $l_{y}$ are very short is also interesting to explore. To do this we treat the autocorrelation function as a Dirac delta function in $x$ and $y$,

$$
U\left(x, x^{\prime}, \bar{y}\right)=4 l_{x} l_{y} \delta\left(x-x^{\prime}\right) \delta(\bar{y})
$$

Rather than using Eq. (31), we go back to Eq. (30) and separate out the dependencies of $\bar{z}$ and $\tau$. After some straightforward calculations it can be shown that in the strong turbulence regime the dimensionless bound $y$ scales as

$$
\gamma \sim(\text { constant }) l_{x} l_{y} R v^{2}
$$


and scales in the quasilinear regime as

$$
\gamma^{-1} \sim(\text { constant })\left(l_{x} l_{y} v\right)^{-1}\left[(K v)^{-1}+l_{z}^{-1}\right]
$$

For finite $l_{z}, \mathrm{Eq}$. (31) can be solved numerically. Since the solutions must resolve two disparate spatial scales, $l_{z}$ and $R^{-1}$, straightforward computational schemes access only a limited range of $l_{x}$ and $R$. For large Reynolds number the asymptotic solution is not readily available analytically; however, it is clear that a finite bound exists for infinite $R$. The reason is that a finite bound for infinite l's and $R$ can be shown to exist, and the bounds decrease with $l_{x}, l_{y}$, and $l_{z}$. Fig. 2 shows that the bounds are smaller for smaller perpendicular correlation length.

\section{CONCLUSION}

In conclusion, we have considered various aspects of the theory of upper bounds for turbulent transport in passive advection problems. Firstly, we have shown how one can generate better bounds for the reference model of Krommes and Smith by including more constraints. (This is, of course, not a new result philosophically; such improvement was already demonstrated in the original work by Howard. ${ }^{(6)}$ The present work simply adds to one's repertoire of examples.) When we consider the constraint which includes information up to and including the triplet cross-correlation, the improvement over the bound of Krommes and Smith is not substantial. Moreover, there is no improvement at all in the strong turbulence regime. When we add a constraint including fourth-order cross-correlations, the bounds are improved substantially in the cases of both finite and infinite Kubo number. Nevertheless, it is quite apparent that such higher-order calculations become rapidly substantially involved, even in the simple one-dimensional reference model where one has the freedom to choose appropriate statistics for the correlation of the advecting velocity field $u$. Thus the utility of bounds invoiving the fourth-order moments for complicated practical self-consistent situations is unclear. However, in some problems where accuracy can be sacrificed in favor of crude estimates, the optimum theory with one or two constraints which recognize the effects of finite correlation time is a promising candidate for the prediction of the steady-state flux.

Secondly, we showed how to solve numerically the reference modei for finite Kubo number. This problem is not entirely trivial due to statistical noise. For the present problem, refined calculations of the exact solution are quite long and expensive relative to the computation of even the most complicated bound.

Finally, we applied the optimum theory to the problem of transport in a collisionless plasma due to stochastic magnetic fields. In doing this, we encountered no untoward difficulty in proceening even when the velocity field depends on space as well as on time. We recovered already known scaling laws of the diffusion coefficient in both the quasilinear regime and the strong turbulence regime when the perpendicular correlation length is 
infinite. Also, we could generate numerically a family of curves of the bounds as a function of the correlation lengths, correlation time, and the Reynolds number.

In closing, we point out that to our knowledge all of the applications thus far have been to inhomogeneous problems. Recently, Kraichnan has argued ${ }^{(17)}$ that such methods can be extended to homogeneous problems as well, and to lower as well as to upper bounds. This raises the exciting possibility of rigorous bounds on the Kolmogorov constant, for example, and suggests that the bounding theory is a fruitful area for further research.

\section{APPENDIX A. NUMERICAL SOLUTION OF THE REFERENCE MODEL}

Here we describe direct numerical solutions of the generalized reference model ( 1 ), together with the boundary conditions

$$
T(0)=1, \quad T(1)=0
$$

The general procedure is as follows. First, we choose an arbitrary initial condition $T(x, 0)$, which we shall take to be the laminar profile

$$
T_{\operatorname{lam}}(x)=1-x
$$

Next, we integrate many realizations sufficiently long that the low-order statistics such as $\langle T\rangle$ become stationary. Finally, we compute the advective contribution $\bar{\Gamma}$ to the flux by averaging over the realizations. As Krommes and Smith discussed, $\bar{\Gamma}$ may be obtained from the gradient at either wall by subtracting off the classical part $R^{-1}$ :

$$
\cdot \vec{\Gamma}=-R^{-1}\left[(T)^{\prime}(\text { wail })+1 !\right. \text {. }
$$

The random field $u(t)$ is ass:imed to be a stationary, centered Gauss-Markov process, which is thus fully specified by its autocorrelation function

$$
U(\tau) \doteq\langle\delta u(\tau) \delta u(0)\rangle=\exp (-|\tau| / K)
$$

Here $K$ is the Kubo number and plays the role of the autocorrelation time $\tau_{a c}$. To obtain realizations of $u(t)$, we generate numerically an Ornstein-Uhlenbeck process, ${ }^{(18)}$ which obeys

$$
\frac{d u}{d t}+\nu u=a(t)
$$

where $\nu=\tau_{a c}^{-1}=K^{-1}$ and $a(i$, is Gaussian white noise. The strength $A$ of $a$, as in

$$
\left\langle\delta a(\tau) \delta a\left(\tau^{\prime}\right)\right\rangle=A \delta\left(\tau-\tau^{\prime}\right),
$$


can be determined from the result

$$
\begin{aligned}
U\left(t, t^{\prime}\right) & =\left(\frac{A}{2 \nu}\right)\left(e^{-\nu t-t^{\prime}}-e^{-\nu\left(t+t^{\prime}\right)}\right) \\
& \rightarrow\left(\frac{A}{2 \nu}\right) e^{-\nu \cdot t-t^{\prime}} \quad\left(t, t^{\prime} \gg T_{\text {ac }}=\nu^{-1}\right)
\end{aligned}
$$

by comparing Eq. (35b) with Eq. (33), we find $A=2 \nu$. However, we never need to generate a white noise process explicitly since in a finite-difference scheme only the time integral of a enters. That is, from Eq. (34)

$$
u(t+\Delta t)=e^{-v \Delta t} u(t)-\Delta u(t)
$$

where

$$
\Delta u(t)=\int_{t}^{t+\Delta t} d t^{\prime} e^{-\nu\left(t+\Delta t-t^{\prime}\right)} a\left(t^{\prime}\right) .
$$

The increment $\Delta u(t)$ is a centered Gaussian since $a$ is; it is also independent of $\Delta u$ in any ather time interval. Thus we require just the variance of $\Delta u$, which can be obtained from Eq. (35a) by replacing both $t$ and $t^{\prime}$ by $\Delta t$ :

$$
\sigma^{2} \doteq\left\langle\Delta u(t)^{2}\right\rangle=1-e^{-2 \nu \Delta t}
$$

[In the short-time limit this reduces correctly to the far-iliar result of velocity-space diffusion,

$$
\left.\sigma^{2} \rightarrow 2 \nu \Delta t \quad(\nu \Delta t \ll 1) \cdot\right]
$$

Thus the final algorithm for $u(t)$ is Eq. (36), with

$$
\Delta u(t)=\sigma \gamma(t)
$$

$\gamma(t)$ being a normal random variable with zero mean and unit variance. Computer routines which generate such numbers are readily available. A typical realization of $u(t)$ is sbown in Fig. 3. The circles indicate the points computed explicitly by the previous algorithm; the solid line is generated by quasi-cubic Hermite interpolation (which allows a possibly discontinuous second derivative) with three segments between each circle. The mean value of $Z=5000$ realizations is also shown as the dotted line. In Fig. 4 we demonstrate that the generated realizations have the proper autocorrelation function. The horizontal dotted lines show the first few e-foldings $e^{-n}(n=1,2,3,4)$; the exact solution $\exp (-\tau / K)$ is the diagonal dotted line. For $Z=1000$ one sees that the result is good through about two e-foldings, after which statistical noise sets in; for $Z=5000$ (not shown) the result is good through about three e-foldings.

We relied on a commercial integration package from the International Mathematical Subroutine Library (IMSL) to integrate Eq. (1) forward in time for each realization $u$, in 
order to most simply maintain careful control of the global integration error. In space. the solutions exhibit boundary layers of width $R^{-1}$ at the walls. (4) Thus we emploved a nonuniform mesh, with $B$ points spaced logarithmically within each boundary laver and $B$ more spaced uniformly between the edge of the boundary laver and the center. In other words, there were $4 B$ mesh points including the walls. Even $B=2$ appeared to be adequate for most calculations; however, for the runs with $Z \geq 1000$ we used $B=3$. Probably a small improvement in accuracy would result from a larger $B$. However, the integration scheme was fourth order in the average mesh spacing, and because the runs with large $Z$ were expensive and are used only to verify that the computed bounds indeed lie above the exact solution, it was not deemed important to make more refined runs.

A typical result for the flux (averaged over all realizations) is shown in Fig. 5 as a lunction of time. An initial transient period is evident during which the mean profile $\langle T\rangle$ evolves from its initial laminar form (with no advertive $f(u x$ ) to its final steady-state value. Curves $L$ and $R$ represent the fux computed from the slopes at the left- and right-hand walls, respectively. Although those values should be equal theoretically by symmetry and should be constant in the long-time limit, they differ and fluctuate in time because of statistical noise due to the finite number of realizations. Curve $\mathrm{M}$ is the average of curves $L$ and $R$ and exhibits reduced statistical fluctuations. The total integration time was determined by trial and error. It was intended to be at least three times the transient period; that, typically, was several autocorrelation times. The final values for the flux were determined from the mean of the time series of curve $M$ during the final two-thirds of the time integration and are indicated by the solid horizontal lines. The results of several such runs are plotted for $R=10$ in Fig. 1 as the solid circles, with error bars determined by the sample standard deviations from the means. There are points with $Z=1000$ for $K=1$, $K=3$, and $K=5$. Each of these runs took about one hour of CPU time on the CRAY-2. (This time could have been reduced significantly by hand-coding the time integration instead of relying on the IMSL routine; however, we opted for reliability and significantly reduced debugging time.) The point at $K=3$ for $Z=1000$, with $\bar{\Gamma}=0.272 \pm 0.013$ is almost overlaid with the result of a run for $Z=5000$, which predicted $\bar{\Gamma}=0.277 \pm 0.006$ (and $L \approx R \approx M$ )-in accord with the prediction of fluctuation theory that the errors scale with $Z^{-1 / 2}$. In Fig. 6 we show the mean profile at the final integration tine (solid line) and at the next-to-last time (dashed line, almost obscured by the solid line). The diagonal dotted line is the laminar profile. The curved dotted line shows a typical realization of the profile in the statistically steady state (this curve fluctuates in time around the laminar profile); the crosses indicate the mesh points. The horizontal and vertical dotted lines are intended to display any deviation of symmetry around the center. An insignificant deviation is evident for $Z=1000$; for $Z=5000$ (not shown), such a deviation is not visible at all. 


\section{APPENDIX B. THE FUNICTIONAL DERIVATIVE ON BOUNDED DOMAINS}

Because we work on a bounded domain in $x$, the final form of the Euler-Lagrange equations depends on whether one works on the open interval $(0,1)$ or on the closed interval $\{0,1]$. In particular, when one works on $[0,1]$, the functional derivative gives rise to delta functions on the boundaries, which may appear difficult to deal with. Thus we here discuss this issue in more detail.

Consider the functional

$$
F(f) \doteq \frac{1}{2} \int_{0}^{1} d \bar{x}\left(\frac{d f}{d \bar{x}}\right)^{2}
$$

where $f(x)$ vanishes at $x=0$ and $x=1$. The functional derivative becomes

$$
\begin{aligned}
\frac{\delta \mathcal{F}}{\delta f(x)} & =-\int_{0}^{1} d \bar{x} f^{\prime \prime}(\bar{x}) \delta(x-\bar{x})+\left.f^{\prime}(\bar{x}) \delta(x-\bar{x})\right|_{\mid z=0} ^{1} \\
& =-[H(x)-H(x-1)] f^{\prime \prime}(x)-f^{\prime}(x)[\delta(x)-\delta(x-1)] \\
& =-f_{+}^{\prime \prime}(x)
\end{aligned}
$$

where we have introduced the Heaviside function $H(x)$ and defined the bounded function $f_{+}(x)$ as

$$
f_{+}(x) \doteq[H(x)-H(x-1)] f(x) .
$$

Thus, if we work on $(0,1)$, the delta functions do not appear explicitly.

As a second example, consider the functional

$$
\mathcal{G}\{f\}=\int_{0}^{1} d \bar{x} \lambda(x) f^{\prime \prime}(\bar{x}) .
$$

The functional derivative is

$$
\begin{aligned}
\frac{\delta G}{\delta f(x)} & =\int_{0}^{1} d \bar{x} \lambda^{\prime \prime}(\bar{x}) \delta(x-\bar{x})+\left.\lambda(\bar{x}) \delta^{\prime}(x-\bar{x})\right|_{t=0} ^{1}-\left.\lambda^{\prime}(\bar{x}) \delta(x-\bar{x})\right|_{t=0} ^{1} \\
& =[H(x)-H(x-1)] \lambda^{\prime \prime}(x)+2 \lambda^{\prime}(x)[\delta(x)-\delta(x-1)]+\lambda(x)\left[\delta^{\prime}(x)-\delta^{\prime}(x-1)\right] \\
& =\lambda_{+}^{\prime \prime}(x) .
\end{aligned}
$$

The functionals $\mathcal{F}$ and $\mathcal{G}$ are two generic types of the functional $\boldsymbol{R}$ in Sec. 2 . Hence, it is clear that the resulting Euler-Lagrange equation in Sec. $\&$ is defined on the open interval $(0,1)$. 


\section{APPENDIX C. OPTIMUM EQUATION FOR TRANSPORT DUE TO STOCHASTIC MAGNETIC FIELDS}

Here we derive a variational principle and associated Euler-Lagrange equation for the problem of collisionless particle transport due to specified stachastic magnetic fields, using a constraint which includes two-point correlations in both space and time. We begin with the modul equation derived in Sec. 3 :

$$
\frac{\partial f}{\partial t}+v \frac{\partial f}{\partial \ell}+\delta u \cdot \nabla f-R^{-1} \nabla_{-}^{2} f=0
$$

where all symbols have been defined in the text. First, we ensemble-average Eq. (37) assuming that the turbulence is statistically stationary in time and homogerieous in the $y$ and $z$ directions. We obtain the continuity equation

$$
\frac{d}{d x}\left\langle\delta u_{x} \delta f\right\rangle-R^{-1} \frac{d^{2}}{d x^{2}}\langle f\rangle=0
$$

Upon integiating once over $r$, we are led to

$$
\frac{d}{d x}\langle f\rangle(x)=R\left[\Gamma_{v}(x, x, 0)-\bar{\Gamma}_{v}(0)-R^{-1}\right]
$$

where we have introduced the two fluxes

$$
\Gamma_{v}\left(x, x^{\prime}, y-y^{\prime}, z-z^{\prime}, t-t^{\prime}\right) \doteq\left\langle\delta f(x, y, z, t) \delta u_{z}\left(x^{\prime}, y^{\prime}, z^{\prime}, t^{\prime}\right)\right\rangle
$$

and

$$
\bar{\Gamma}_{v}(y, z, t) \doteq \int_{0}^{t} d x \Gamma_{v}(x, x, y, z, t)
$$

For convenience, when no ambiguity arises we write 0 as a shorthand for $(y, z, t)=(0,0,0)$, as in $\bar{\Gamma}_{v}(0)$ in Eq. (39). The equation for the fluctuation is

$$
\left(\frac{\partial}{\partial t}+v \frac{\partial}{\partial \ell}\right) \delta f+\delta u_{x} \frac{d}{d x}\langle f\rangle+\nabla \cdot(\delta u \delta f-\langle\delta u \delta f\rangle)-R^{-1} \nabla_{\perp}^{2} \delta f=0
$$

where $\nabla \cdot \delta u=0$ was used.

We now proceed to derive the basic constraint. Upon multiplying Eq. (40) by $\delta f$ at the same point in space and time, we obtain

$$
\Gamma_{v}(x, x, 0) \frac{d}{d x}\langle f\rangle+\frac{d}{d x}\left\langle\frac{1}{2} \delta u_{x} \delta f^{2}\right\rangle-R^{-1}\left\langle\varepsilon_{J} \nabla_{\perp}^{2} \delta f\right\rangle=0
$$

Upon using $\mathrm{Eq}$. (39) to eliminate $d(f) / d x$, integrating over $x$, and rearrangii.g, we are led to the basic constraint

$$
\bar{\Gamma}_{v}(0)=R \overline{\Delta \Gamma_{v}^{2}}+R^{-1} \overline{\left.\left(\nabla_{\perp} \delta f\right)^{2}\right\rangle}
$$


where

$$
\Delta \Gamma_{v}(x) \doteq \Gamma_{v}(x, x, 0)-\bar{\Gamma}_{v}(0)
$$

Thus the basic variational principle is

$$
\text { minimize } R=\gamma_{*} \frac{R^{2} \overline{\Delta \Gamma_{z}^{2}}-\overline{\left.\left(\nabla \sum \delta f\right)^{2}\right)}}{\bar{\Gamma}_{v}(0)^{2}} \text { subject to } \bar{\Gamma}_{v}(0)=\gamma_{\nabla} \text {. }
$$

To refine the bounds by including the coherence properties of the advecting stochastic field, we must use a two-point constraint. Krommes and Smith showed how to do this when the advecting field depended only on time, but bere we wish to consider space dependence as well. The procedure is as follows. First, we multiply Eq. (+40) by $6 u_{z}\left(x^{\prime}, y^{\prime}, z^{\prime}, t^{\prime}\right)$ and ensemble-average:

$$
\begin{aligned}
& 0=\left(\frac{\partial}{\partial \tau}-v \frac{\partial}{\partial \hat{\ell}}\right) \Gamma_{y}\left(x, x^{\prime}, \bar{y}, \bar{\Sigma}, \tau\right)+U\left(x, x^{\prime}, \bar{y}, \bar{z}, \tau\right) \frac{d}{d x}(f)(x) \\
& +\nabla \cdot T\left(x, x^{\prime}, \bar{y}, \bar{j}, \tau\right)-R^{-1} \nabla_{-}^{2} \Gamma,\left(x, x^{\prime}, \bar{y}, \bar{j}, \tau\right) \text {, }
\end{aligned}
$$

where $\bar{y}, \equiv$, and $t$ are the difference variables $\bar{y} \doteq y-y^{\prime}, \equiv \doteq z-z^{\prime}$, and $\tau \doteq t-t^{\prime}, U$ is the autocorrelation function of $u_{x}$, and $T$ is a triplet cross-correlation function:

$$
\begin{aligned}
& U\left(x, x^{\prime}, \bar{y}, \bar{\Sigma}, \tau\right) \doteq\left(\delta u_{x}(x, y, z, t) \delta u_{x}\left(x^{\prime}, y^{\prime}, z^{\prime}, t^{\prime}\right)\right\rangle, \\
& T\left(x, x^{\prime}, \bar{y}, \bar{z}, \tau\right) \doteq\left(\delta u(x, y, z, t) \delta f\left(x, y, z^{\prime}, t\right) \delta u_{z}\left(x^{\prime}, y^{\prime}, z^{\prime}, t^{\prime}\right)\right) .
\end{aligned}
$$

After we integrate over $x, x^{\prime}$, and $\bar{y}$, we immediately obtain the two-point constraint

$$
\begin{aligned}
0=C(\Sigma, \tau) \doteq & \int_{-\infty}^{\infty} d \bar{y} \int_{0}^{l} d x d x^{\prime}\left(\frac{\partial}{\partial \tau}+v \frac{\partial}{\partial \bar{z}}-R^{-1} \frac{\partial^{2}}{\partial x^{2}}\right) \Gamma v\left(x, x^{\prime}, \bar{y}, \bar{\Sigma}, \tau\right) \\
& -\int_{-\infty}^{\infty} d \bar{y} \int_{0}^{1} d x^{\prime} U\left(0, x^{\prime}, \bar{y}, \bar{z}, \tau\right),
\end{aligned}
$$

where we used the divergence-fice property of dec to write

$$
U\left(x, x^{\prime}, \bar{y}, \bar{z}, \tau\right) \frac{d}{d x}(f)(x)=\nabla \cdot\left[\left(\delta u \delta u_{x}^{\prime}\right)(f)\right]
$$

and where the boundary conditions were used to obtain the last tertn of Eq. (45). (Notice that the effect of magnetic shear is lost under the $\bar{y}$ integration.)

Now, we can proceed to construct the functional which is to be minimized:

$$
\begin{aligned}
& R=\gamma_{*} \frac{R^{2} \overline{\Delta \Gamma_{t}^{2}}+\overline{\left.\left(\nabla_{I} \delta\right)^{2}\right\rangle}}{\bar{\Gamma}_{v}(0)^{2}} \\
& +\int_{-\infty}^{\infty} d \tau d \xi \lambda_{0}\left(\overline{z_{1}} \tau\right) C(\bar{\Sigma}, \tau)+\lambda_{1}\left[\Gamma,(0)-\gamma_{z}\right] .
\end{aligned}
$$


In principle, the quantities in this functional should be integrated over $v$ as well. (See the discussion by Krommes and Smith.) However, this is an issue only when collisions are taken into account. Here, since we ansider the collisionless approximation, the velocity rariable enters only as a parameter and we can omit the velocity integration.

The functional derivative of Eq. (16) with respect to of leads to the Euler-Lagrange equation. After using Eqs. (39) and ( 45$)$, we obtain

$$
\begin{aligned}
0=- & R^{-1} \nabla_{\perp}^{2} \delta f+\delta u_{2} \frac{d}{d x}(f)+\Lambda_{1} \delta u_{x} \\
& -\int_{-\infty}^{\infty} d \bar{\gamma} d \bar{s} \Lambda_{0}(\bar{z}, \bar{\tau}) \\
& \times\left(\frac{\partial}{\partial \bar{\tau}}+v \frac{\partial}{\partial \bar{z}}\right) \int_{-\infty}^{\infty} d \bar{y} \int_{0}^{1} d \bar{x} \delta u_{x}(\bar{x}, y-\bar{y},=-\bar{z}, t-\bar{\tau})
\end{aligned}
$$

where

$$
\lambda_{i}=\left(\frac{\gamma_{n}}{2 R}\right) \lambda_{i}
$$

In the same manner as in Sec. $2, \Lambda_{1}$ can be related to $\Lambda_{0}$ by multiplying $\delta f$ at the same point, ensemble-averaging, and integrating over $x$ :

$$
\Lambda_{1}=-\gamma_{0}^{-1} \int_{-\infty}^{\infty} d \bar{\tau} d \bar{z} \Lambda_{0}(\bar{z}, \bar{\tau}) \int_{-\infty}^{\infty} d \bar{y} \int_{0}^{1} d \bar{x} U(0, \bar{x}, \bar{y}, \bar{z}, \bar{\tau}) .
$$

By multiplying Eq. (47) by $\delta t_{x}\left(x^{\prime}, y^{\prime}, z^{\prime}, t^{\prime}\right)$ and averaging, the Euler-Lagrange equation can be rewritten as an equation for the flux itself;

$$
\begin{aligned}
0=- & R^{-1} \nabla_{\perp}^{2} \Gamma_{s}\left(x, x^{\prime}, \bar{y}, \bar{z}, \tau\right)+U\left(x, x^{\prime}, \bar{y}, \bar{z}, \tau\right) \frac{d}{d x}(\rho) \\
& -\left(\frac{\partial}{\partial \tau}+v \frac{\partial}{\partial \bar{z}}\right) \phi\left(x^{\prime}, \bar{z}, \tau\right)-\gamma_{\nu}^{-1} \phi(0) U\left(x, x^{\prime}, \bar{y}, \bar{z}, \tau\right),
\end{aligned}
$$

where $\phi$ is the convolution of $\Lambda_{0}$ and $U$ :

$$
\phi(x, \bar{z}, \tau) \doteq \int_{-\infty}^{\infty} d \bar{\tau} d \bar{z} \Lambda(\bar{z}, \bar{\tau}) \int_{-\infty}^{\infty} d \bar{y} \int_{0}^{1} d \bar{x} U(x, \bar{x}, \bar{y}-\bar{y}, \bar{z}-\bar{z}, \tau-\bar{\tau}) .
$$

Notice that $\phi$ is not a function of $\tilde{y}$. In order to deduce the relation between $\phi$ and $\Gamma_{\mathrm{y}}$, we integrate Eq. (48) over $x, x^{\prime}$, and $\bar{y}$. Since it is assumed that the $\bar{y}$ integrations of $\Gamma$. and $U$ are finite, and since the integral of $\phi$ over $\bar{y}$ is infinite, in order for Eq. (48) to be satisfied we must let the third term on the right-hand side of $\mathrm{Eq}$. (48) vanish:

$$
\begin{gathered}
\left(\frac{\partial}{\partial t}+v \frac{\partial}{\partial \bar{z}}\right) \phi=0 . \\
-27-
\end{gathered}
$$


This leads to

$$
\phi(x, \bar{z}, \tau)=\phi(x, \bar{z}-v \tau) .
$$

After we use the constraint $(45), \phi(0)$ is found from the integration of Eq. (48) to be

$$
\phi(0)=-\gamma_{0}\left[\int_{-\infty}^{\infty} d \bar{y} \overline{\bar{U}}(\bar{y}, \bar{z}, \tau)\right]^{-1}\left(\frac{\partial}{\partial \tau}+v \frac{\partial}{\partial \bar{z}}\right) \int_{-\infty}^{\infty} d \bar{y} \overline{\bar{\Gamma}}_{\mathrm{v}}\left(\bar{y}_{\mathrm{i}} \bar{z}, \tau\right),
$$

where ue defined double-barred quantities as

$$
\overline{\bar{f}} \doteq \int_{0}^{1} d x d x^{\prime} f\left(x, x^{\prime}\right)
$$

The above suggests that $\Gamma_{\nabla}$ and the correlation function $U$ have the same form as a function of $\Sigma$ and $\tau$. Finally, after we substitute for $\phi(0)$, we have

$$
\begin{aligned}
0=- & R^{-1} \nabla_{\perp}^{2} \Gamma_{v}\left(x, x^{\prime}, \bar{y}, \bar{z}, \tau\right)+R\left[\Gamma_{v}(x, x, 0)-\bar{\Gamma}_{v}(0)-R^{-1}\right] U\left(x, x^{\prime}, \bar{y}, \bar{z}, \tau\right) \\
+ & {\left[\int_{-\infty}^{\infty} d \bar{y} \overline{\bar{U}}(\bar{y}, \bar{z}, \tau)\right]^{-1} } \\
& \times\left[\left(\frac{\partial}{\partial \tau}+v \frac{\partial}{\partial \bar{z}}\right) \int_{-\infty}^{\infty} d \bar{y} \overline{\bar{\Gamma}}_{v}(\bar{y}, \bar{z}, \tau)\right] U\left(x, x^{\prime}, \bar{y}, \bar{z}, \tau\right) .
\end{aligned}
$$

This equation is discussed further in Sec. 3 .

\section{REFERENCES}

1. R. H. Kraichnan, J. Math. Phys. 2:124 (1961).

2. J. A. Krommes, in Basic Plasma Physics II, edited by A. A. Galeev and R. N. Sudan (North-Holland, Arnsterdam, 1984), Chap. 5.5, p. 183.

3. F. H. Busse, Adv. Applied Mech. 18:77 (1978),

4. J. A. Krommes and R. A. Smith, Ann. Phys. 177:246 (1987).

5. W. V. R Malkus, Proc. Roy. Soc. A 225:196 (1954).

6. L. N. Howard, J. Fluid Mech. 17:405 (1963).

7. R. A. Smith, Ph.D thesis, Princeton University, 1986.

8. R. Kubo, J. Math. Phys. 4:174 (1963),

9. T. H. Stix, Phys. Rev. Lett. 30:833 (1973). 
10. A. B. Rechester and M. N. Rosenbluth, Phys. Rev, Lett. 40:38 (1978).

1]. J. A. Krommes, C. Oberman, and R. H. Kleva, J. Plasma Phys. 30:11 (1983).

12. P. W. Terry, P. H. Diamond, and T. S. Hahm, Phys. Rev. Lett. 57:1899 (1986)

13. J. A. Krommes and C.-B. Kim, Phys. Fluids 31:869 (1988).

14. A. A. Thoul, P. L. Similon, and R. N. Sudan, Phys. Rey. Lett. 59:1448 (1987).

15. J. R. Jokipii and E. N. Parker, Astrophys. J. 155:777 (1969).

16. B. B. Kadomtsev and O. P. Pogutse, in Plasma Physics and Controlled Nuclear Fusion Reseach 1978 (IAEA, Vienna, 1979), p. 649.

17. R. H. Kraichnan, U.S.-Japan Workshop on Plasma and Fluid Turbulence (Austin, TX, December 1987); private communication (1987).

18. See, for example, J. M. Sancho, M. san Miguel, S. L. Katz, and J. D. Gunton, Phys. Rer. A 26, 1589 (1982); R. F. Fox, R. Roy, and A. W. Yu, J. Stat. Phys. 47, 47t (1987). 


\section{FIGURE CAPTIONS}

Figure 1. Various upper bounds on $\bar{\Gamma}$ for the reference model as a function of Kubo number $K$, for Reynolds numbers $R=5,10$, and 50 . See the text for definitions of the various curves. The solid circles with error bars are the "exact" results. from numerical integration of many realizations. The arrows indicate the exact results for $K=\infty$.

Figure 2. Bounds for the problem of stachastic magnetic fields for two perpendicular correlation lengths $l_{z}=l_{y}=2$ and 50 as a function of the parallel correlation length $l_{\text {; }}$ when $K=100$ and $R=5$.

Figure 3. A typical realization of the Ornstein-Uhlenbeck process $u(t)$ for $K=3$.

Figure 4. Comparison of the numerically generated autocorrelation function with the expected function $\exp (-\tau / K) . K=3, Z=1000$.

Figure 5. Numerical solution for $\bar{\Gamma}(t)$ at $K=3$, computed with $Z=1000$ from the slope of $\langle T\rangle$ at the left wall (L), the right wall (R), and the mean $M$ of $L$ and $R$. The time averages of the last two-thirds of the time series are shown as the solid horizontal lines.

Figure 6. For $Z=1000$, the mean profile at the final time (solid line) and a typical realization (curved dotted line). The crosses indicate the spatial mesh. See text for further discussion. 
$88 T 1032$

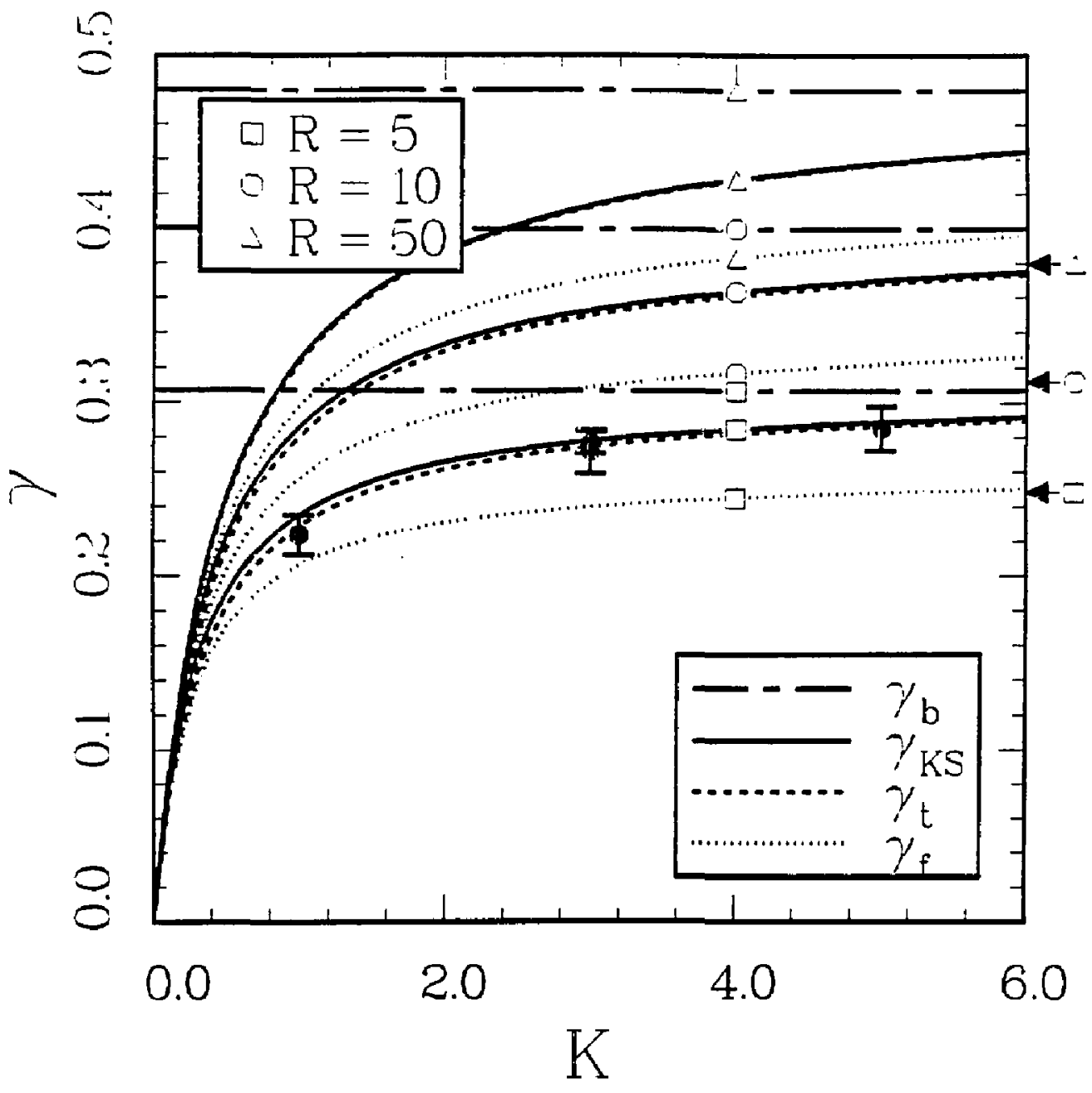

Fig. 1 
88T1033

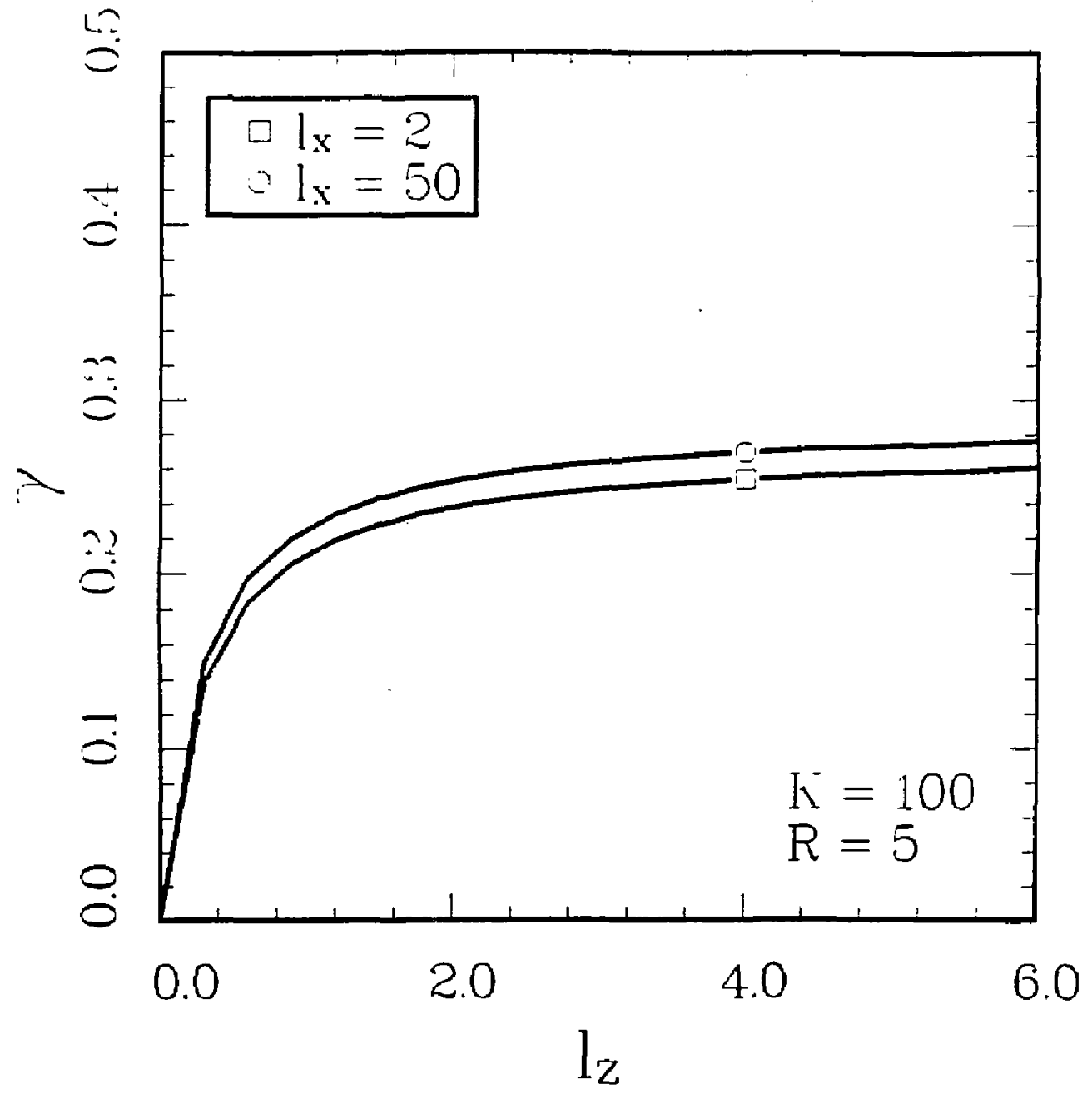

Fig. 2

$-32-$ 
88T 1034

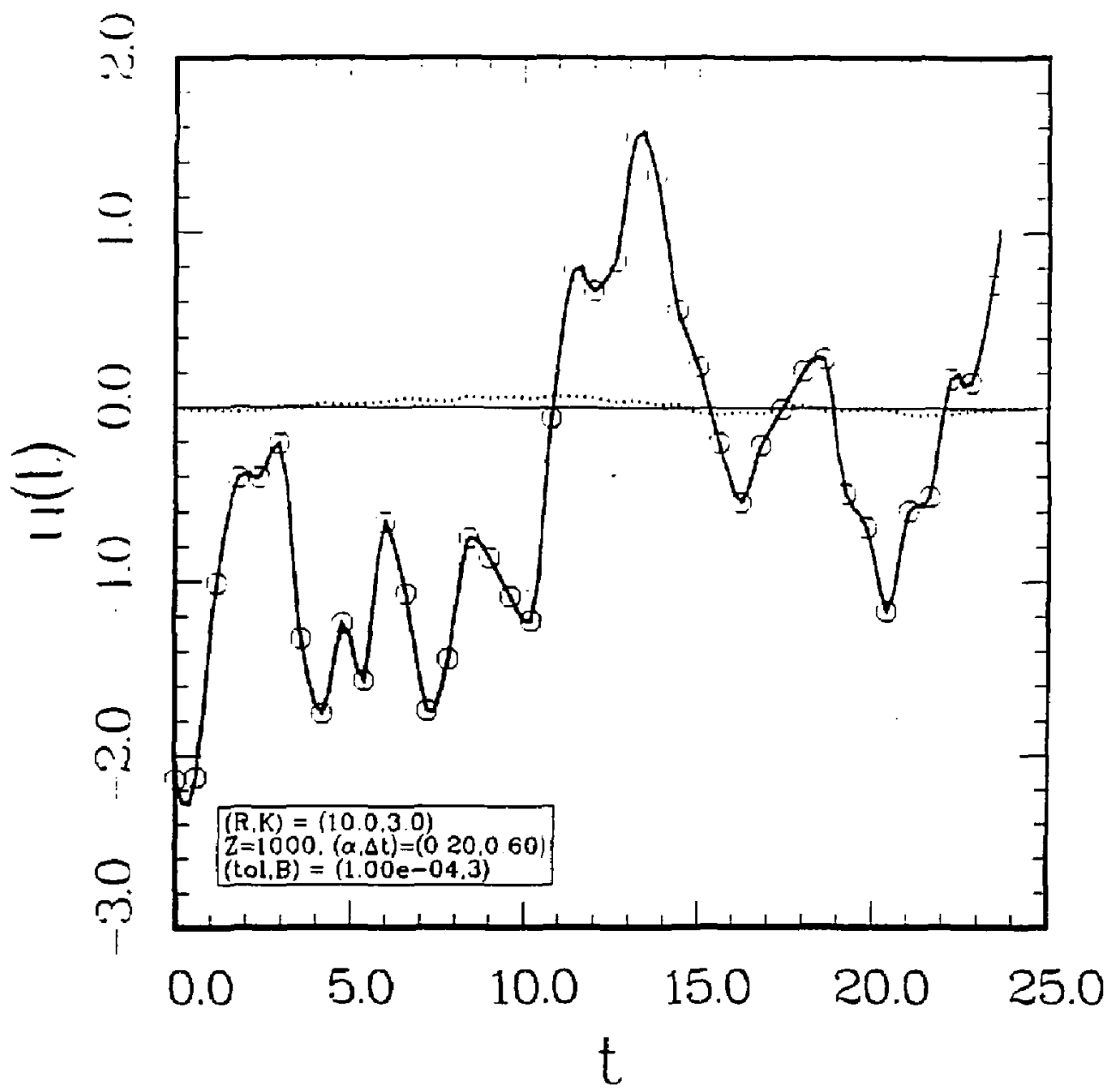

Fig. 3 


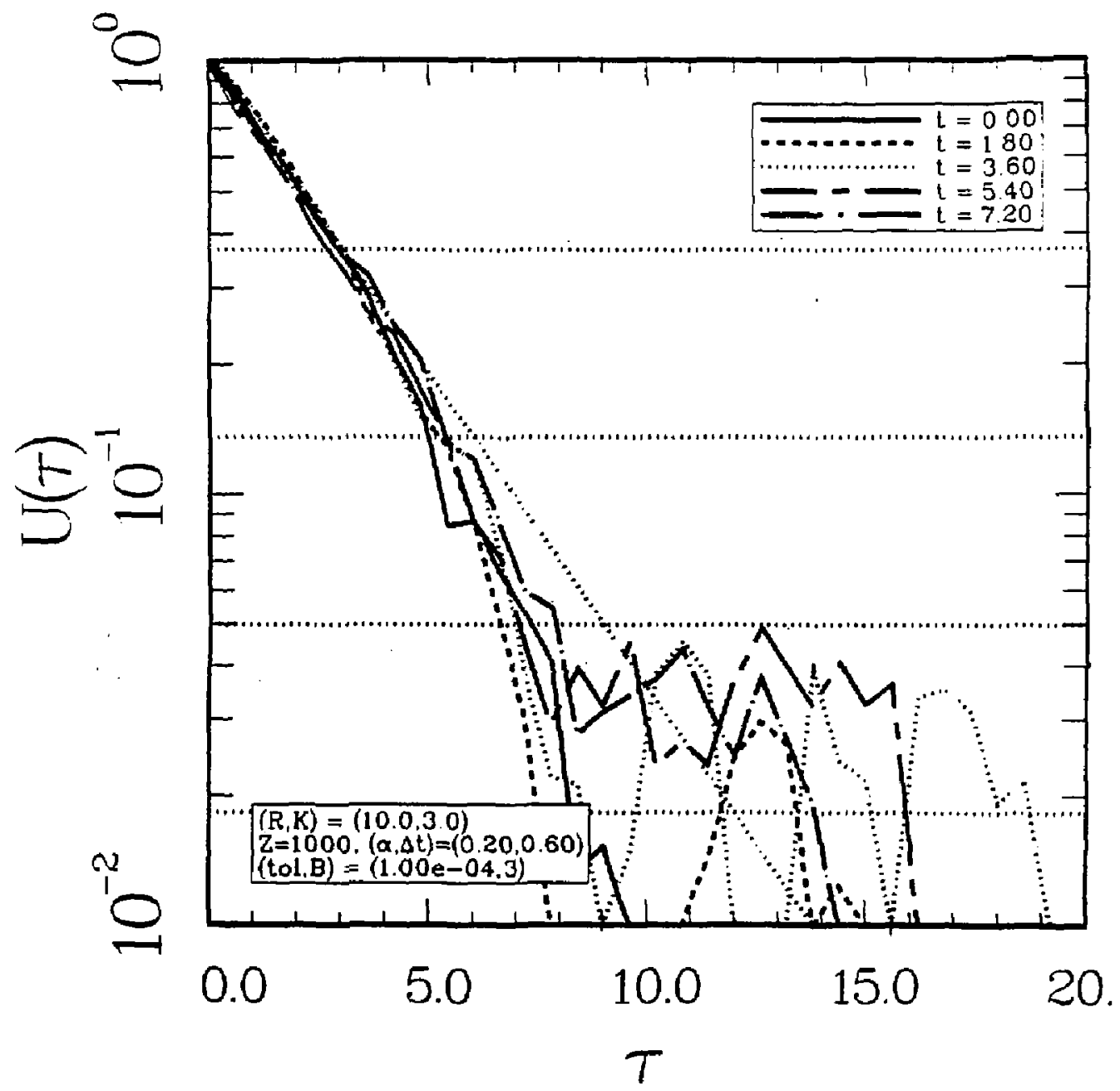

Fig. 4 
$88 T 1036$

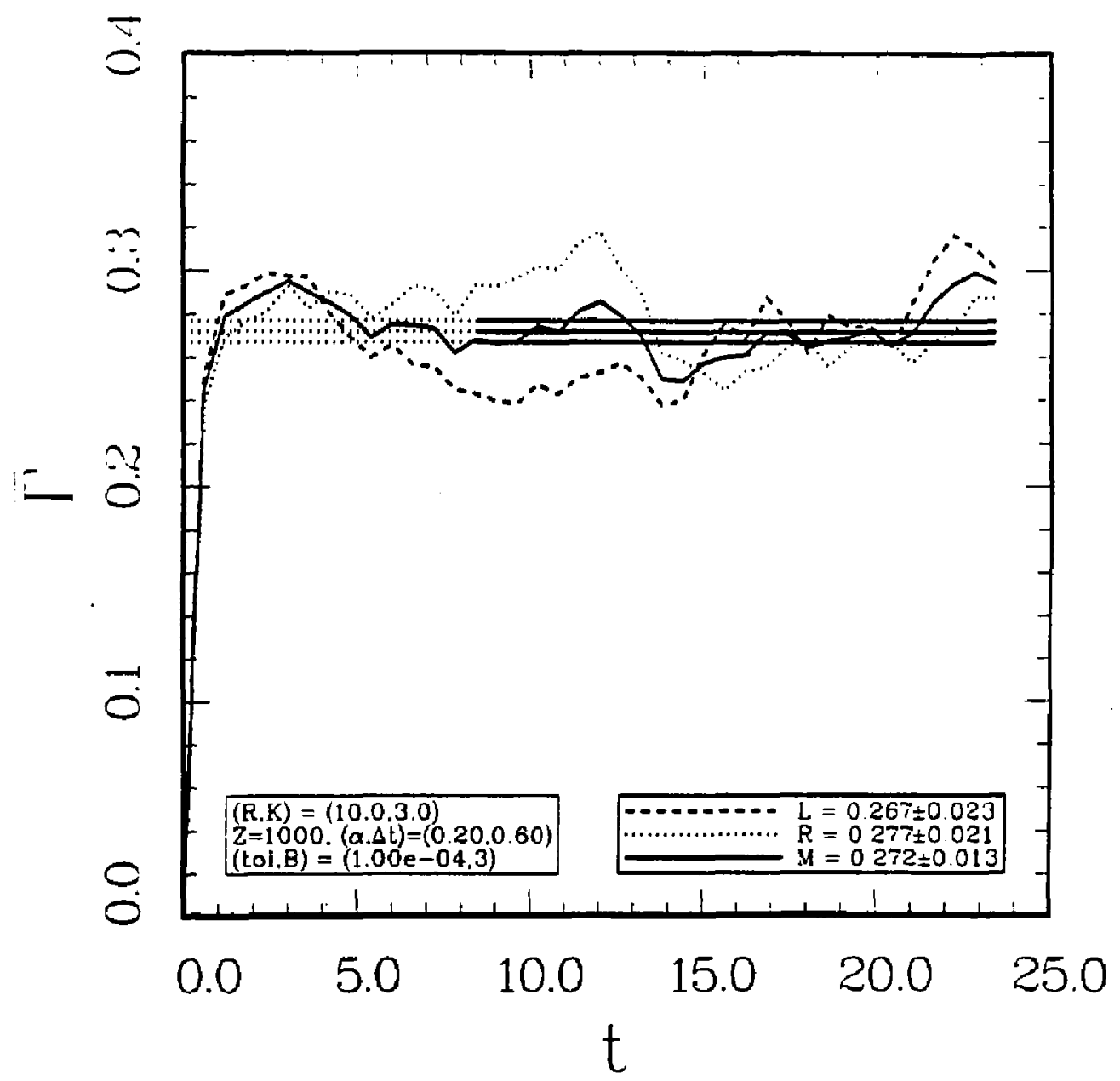

Fig. 5 
88T1037

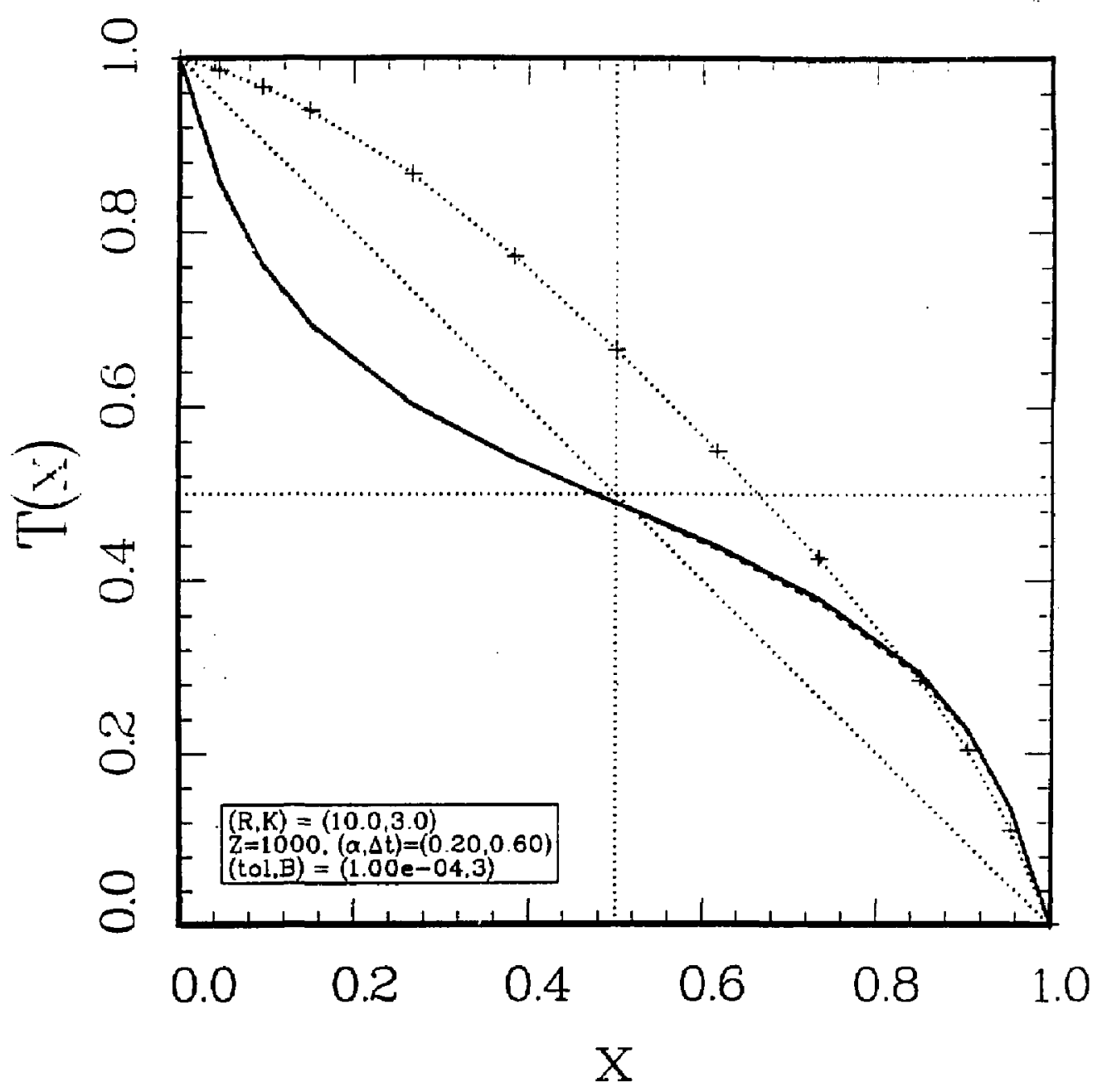

Fig. 6 
Or. Frank J. Paoloni, Univ of Wollongong, Austral, la Prot. M.M. Erennan, Univ Sydney, Australia

Plassa Researeh Lad., Australian Nat. Univ., AUSTRALIA

Prot. I.R. Jones, Filinders Univ., AuSTRALIA

Prot. F. Coo, inst Theo Phys, AUSTRia

Prot. M. Heineler, Institut i ur Theoretische Physix, AustR!A

M. Coossens, Astronomisch Instituut, aELGiUM

Ecole Royale militaire, Lab da Phys Plasmas, BELGiUm Comissian-Eurogean, Og-XII fusion Prog, BELGium

Prot. a. Bouclaue, Laborator i um iecr Natuurkunde, BELGILM

Dr, P.H. Sakanaka, Instituto Fisica, SRAzill

Instiruto de Pescuisas Esoaciasi-INPE, BRAzIL

Dacuments off ice, Atomic Energy of Canada Limited, CanaDA

Dr. M.P. Bachynski, upg Toehnologies, Ine., CANAOA

Dr. H.M. Skarsgard, University of Soskatehewan, CANADA

Dr. H. Garnard, University of Britigh Columbia, CANADA

Prot. L. Teichmann, univ. of Montrear, CANAOA

Prot. S.R. Sreenirasan, University of Calgary. CANADA

Prof. Tudor w. Jornston, INAS-Energie, CANADA

Dr. C.R. James, H.iv, of Alberta, CANMDA

Jr. Perer Lukac, Momenskeho Univ. CzEchoslovakta

The Librarian. Cuinam Laboratory. ENGLAND

The Librarian, Ruthertord ADpleton Laboratory, EMGLAND

Mrs. S.A. Hutchinson, JET Library, ENGLAND

C. Mouttet, Lab. de Physique des Milioux Ionisés, FRANCE

J. Radet, CEn/CADARACHE - Bat 506, fRANCE

Univ, of loannina, Library of Physics Dept. GREsCE

Dr. Tom Mual, Academy Bibliographic Ser.. HONG KONG

Prearint Library, Hungarian Meadeny of Sciences, HLNGARY

Or. B. Dasgupta, Sana Inst of Nuet, Phys., INOIA

Or. P. Kaw, Institute for Plasma Rosearch, INOIA

Or. Philip Roserau, israel Inst. Teen, ISRAEL

Librarian, Int'l Cer Theo Phys, ITALy

Prof. G. Rostagni, Univ Oi Pacova, ITAly

Miss Clelia De Palo, Assoc EURATOn-ENEA, ITALY

Biblioteca, Instituto di fisica del PI asma, ITALY

Or. H. Yamato. Toshiva Res d Oev, JaPan

Prof. I, Kawakami, Atomic Energy Res. Instituta, JAPAN

Prof. Kyoji Nishikana, Univ of Hi roshima, JAPAN

Oirec. Dept. Large Tokamak Res. JAERI, JAPAK

Prof. Satoshi Iton, Kyushu University, JAPAN

Resuarch Info Center, Nagoya University, JAPAM

Prof. 5. Tanaka, Kyoto Univarsity, JAPAN

Library, Kyoto University, JAPAN

Prof. NObuyuki InOUE, University of TOKYo, JAPAN

S. Mori, JAERI, JAPAN

Librarian, Kored Advanced Energy Res. Instifute, KOREA

Prof. D.1. Choi, Adv. Inst Sei a Tach, KOREA

Prof. A.s. Lilor, University of Walkato, NEW ZEALND

Institute of Plasma Physics, PEOPLE's REPU⿴囗十 $1 \mathrm{C}$ of CHIM

Librarian, Institute of Phys., PEOPLE's RePLal. IC OF CHINA

Library, Tsing HLo University, FEOPLE'S REPU⿴囗IC OF CHAM
Z. Li, Southwest Inst. Physics, FEOPLE'S REPU⿴LIC GF CHINA Prof. J.A.C. Cabral, Inst Superior Tecnico, PORTUGAL Dr. Oetavian Petrus, al I CuzA University, ROMAHIA Dr. Jahan de villiers, Fusion Studies, AEC, SO AFRICA Prof. M.A. Hellberg, University of Natal, SO LFRICA C.I.E.M.A.T., Fusion Div. Library, SPAIN

Dr. Lennart Stanflo, University of UMEA, SWEDEN

Library, Royal Inst rech, SHEDEN

Prot. Hans Wilhelnson, Chalmers Un iv Teeh, SWEDEN

Centre Phys das Plasmas, Ecole Polytech Fed, SwitZERLAND

Bibliatheek, Fom-Inst joor Plasma-Fysica, THE NETHERL.ANCS

Dr. D.O. Ryutov, Sibarian Aead Sei, LSSR

Or. G.A. Eliseev, Kurcnatov institute, USSR

Dr. V.A. Glukhikn, IAst Electropnysical ADDarjtus, USSR

Or. V.T. Colok, Inst. Phys. Tech. USSR

Or. L.M. Kouriznnykn, Institute Gen. Physics, USSR

Nuclear Res. Establisnment, Julien Lta.. M. GeFMANr

gibliothek, inst. Fur Plasmgtorsenung. W. Gefamany

Or. K. Seningler, Runr Universitat Bochum, A. GEPMANA

ASDEX Ragding Rm, IPo/Max-Planck-1nstitut t $\mathrm{Rr}$

Plasmaphysik. W. GeFHany

Librarian, Max-PIanck Institut, M. GERMANY

Prot. R.K. Janev, Inst Phys, rugoslavia 